\begin{tabular}{|c|c|}
\hline Title & Enhanced Binding in a General Class of Quantum Field Models \\
\hline Author(s) & A rai, A sao; Kawano, Hiroyuki \\
\hline Citation & $\begin{array}{l}\text { Reviews in mathematical physics, 15(4), 387-423 } \\
\text { https://doi.org/10.1142/\$0129055X } 03001680\end{array}$ \\
\hline Issue Date & $2003-06$ \\
\hline Doc URL & http:/hdl .handle.net $/ 2115 / 38257$ \\
\hline Rights & $\begin{array}{l}\text { Electronic version of an article published as Reviews in Mathematical Physics, 15(4), 2003, pp. 387-423, DOI: } \\
10.1142 / \text { : } 0129055 X 03001680 \text { (c) copyright W orld Scientific Publishing Company, } \\
\text { http:/www.worldscinet.com/rmp/rmp.shtml }\end{array}$ \\
\hline Type & article (author version) \\
\hline File Information & ArKa.pdf \\
\hline
\end{tabular}

Instructions for use 


\title{
Enhanced Binding in a General Class of Quantum Field Models
}

\author{
Asao Arai and Hiroyuki Kawano \\ Department of Mathematics \\ Hokkaido University \\ Sapporo 060-0810
}

Japan

E-mail:\{arai,kawano\}@math.sci.hokudai.ac.jp

\begin{abstract}
We consider, in an abstract form, a system of "quantum particles" coupled to a Bose field. It is shown that, under suitable hypotheses, the composed system can have a ground state even if the uncoupled particle system has no ground state.
\end{abstract}

\section{Introduction}

In a quantum system with a coupling parameter $\lambda \in \mathbb{R}$, it may occur that the Hamiltonian of the system has a ground state for a non-zero $\lambda$ even if it has no ground state at the zero-coupling $\lambda=0$. If such a phenomenon occur, then we call it the enhanced binding in the quantum system under consideration.

A typical example is a quantum mechanical system whose Hamiltonian is given by the Schrödinger operator $H_{\mathrm{S}}(\lambda):=-\Delta+\lambda V$ on $L^{2}\left(\mathbb{R}^{d}\right)$, where $\Delta$ is the $d$-dimensional generalized Laplacian and $V: \mathbb{R}^{d} \rightarrow \mathbb{R}$ is a potential. Indeed, it is well known that $H_{\mathrm{S}}(0)$ has no ground state, but, for a general class of $V, H_{\mathrm{S}}(\lambda)$ with $\lambda \neq 0$ has a ground state (e.g., [17]). As a next stage, it is interesting to investigate if enhanced binding occurs in a quantum system of particles coupled to a quantum field.

Recently the study of enhanced binding in non-relativisic quantum electrodynamics (QED) was intiated by Hiroshima and Spohn [14] in the case of the Pauli-Fierz model in the dipole approximation and then by Hainzl, Vougalter and Vugalter [11] in the case of Pauli-Fierz model without the dipole approximation. In [14] it is shown that, under suitable conditions, the enhanced binding occurs for large coupling constants. On the other hand, in [11], the enhanced binding is shown to occur for small coupling constants and for a class of potentials. The results and methods in [11] have been extended to the Pauli-Fierz model with spin [8, 9] (see also [10]).

In this paper we consider enhanced binding in an abstract model of "quantum particles" coupled to a multi-component Bose field. We prove that, under suitable hypotheses, 
enhanced binding occurs in this model. This suggests that enhanced binding in quantum particle-field interaction systems is a general phenomenon, although it may depend on the type of interactions.

The present paper is organized as follows. In Section 2 we describe the model considered and state the main results. The model is essentially same as that discussed in the previous papers $[4,5,6]$ except that the Bose field is a multi-component one. In Section 3 we prove the self-adjointness of the total Hamiltonian of the model, where we present a method different from the one used in $[4,5]$. In considering the problem of enhanced binding in the model, we distinguish two cases: the case where the Bose field is massive and the one where the Bose field is massless, but, without infrared singularity. We first prove the existence of enhanced binding in the massive case. This is done in Section 4. Section 5 is devoted to proof of the existence of enhanced binding in the massless case. In the last section we apply these general results to the Pauli-Fierz type model without $A^{2}$-term in the dipole approximation. In particular, we show that, if the regime of momenta of photons interacting with the quantum particle becomes sufficiently large with an infrared cutoff fixed, then the model has a ground state at least for the coupling constant in some bounded open interval even if the unperturbed particle Hamiltonian has no ground state. The present paper has two appendices. In Appendix A, we formulate, in an abstract form, the weak differentiability of Heisenberg type operators. In Appendix $\mathrm{B}$, we establish, in an abstract framework, a theorem on the existence of a ground state of a self-adjoint operator and a limit theorem of ground states. Each theorem clarifies a general structure underlying methods used in proofs of existence of ground states in non-relativistic QED $[7,12,13]$. These theorems may be interesting also in its own right in the spectral theory of self-adjoint operators.

\section{Definition of the Model and the Main Results}

We consider, in an abstract form, a model of a quantum system $\mathbf{S}$ coupled to a multicomponent Bose field. We denote the Hilbert space of the system $\mathbf{S}$ by $\mathcal{H}$, which is taken to be an arbitrary separable complex Hilbert space. In concrete realizations, $\mathrm{S}$ may be a system of quantum particles or a quantum field system.

In general we denote the inner product and the norm of a Hilbert space $\mathcal{X}$ by $\langle\cdot, \cdot\rangle_{\mathcal{X}}$ and $\|\cdot\|_{\mathcal{X}}$ respectively, where we use the convention that the inner product is antilinear (resp. linear) in the first (resp. second) variable. If there is no danger of confusion, then we omit the subscript $\mathcal{X}$ in $\langle\cdot, \cdot\rangle_{\mathcal{X}}$ and $\|\cdot\|_{\mathcal{X}}$. For a linear operator $T$ on a Hilbert space, we denote its domain by $D(T)$. For a subspace $D \subset D(T), T \mid D$ denotes the restriction of $T$ to $D$. If $T$ is densely defined, then the adjoint of $T$ is denoted $T^{*}$. For linear operators $S$ and $T$ on a Hilbert space, $D(S+T):=D(S) \cap D(T)$ unless otherwise stated.

For a self-adjoint operator $S$ on a Hilbert space, we denote its spectrum (resp. essential spectrum) by $\sigma(S)$ (resp. $\sigma_{\text {ess }}(S)$ ) and its spectral measure by $E_{S}(\cdot)$. If $S$ is bounded from below, then we set

$$
E_{0}(S):=\inf \sigma(S)
$$

the ground state energy of $S$. We say that $S$ has a ground state if $E_{0}(S)$ is an eigenvalue of $S$; in this case, each non-zero vector in $\operatorname{ker}\left(S-E_{0}(S)\right)$ is called a ground state of $S$. 
To describe the Bose field, one uses the Boson Fock space over a separable complex Hilbert space $\mathcal{X}$ :

$$
\begin{aligned}
\mathcal{F}_{\mathrm{b}}(\mathcal{X}) & :=\oplus_{n=0}^{\infty} \otimes_{\mathrm{s}}^{n} \mathcal{X} \\
& =\left\{\psi=\left\{\psi^{(n)}\right\}_{n=0}^{\infty} \mid n \geq 0, \psi^{(n)} \in \otimes_{\mathrm{s}}^{n} \mathcal{X}, \sum_{n=0}^{\infty}\left\|\psi^{(n)}\right\|^{2}<\infty\right\},
\end{aligned}
$$

where $\otimes_{\mathrm{s}}^{n} \mathcal{X}$ denotes the $n$-fold symmetric tensor product of $\mathcal{X}$ with $\otimes_{\mathrm{s}}^{0} \mathcal{X}:=\mathbb{C}$ (the set of complex numbers).

As is well known $[16, \S \mathrm{X} .7]$, one of basic objects on $\mathcal{F}_{\mathrm{b}}(\mathcal{X})$ is the annihilation operator $a(f)(f \in \mathcal{X})$ which is a densely defined closed linear operator on $\mathcal{F}_{\mathrm{b}}(\mathcal{X})$ such that, for all $\psi=\left\{\psi^{(n)}\right\}_{n=0}^{\infty} \in D\left(a(f)^{*}\right),\left(a(f)^{*} \psi\right)^{(0)}=0$ and

$$
\left(a(f)^{*} \psi\right)^{(n)}=\sqrt{n} S_{n}\left(f \otimes \psi^{(n-1)}\right), \quad n \geq 1,
$$

where $S_{n}$ is the symmetrization operator on $\otimes^{n} \mathcal{X}\left(S_{n}^{*}=S_{n}, S_{n}^{2}=S_{n}, \otimes_{\mathrm{s}}^{n} \mathcal{X}=S_{n}\left(\otimes^{n} \mathcal{X}\right)\right)$. The adjoint $a(f)^{*}$, called the creation operator, and the annihilation operator $a(g)(g \in \mathcal{X})$ obey the canonical commutation relations

$$
\left[a(f), a(g)^{*}\right]=\langle f, g\rangle, \quad[a(f), a(g)]=0, \quad\left[a(f)^{*}, a(g)^{*}\right]=0
$$

for all $f, g \in \mathcal{X}$ on the dense subspace

$$
\begin{aligned}
\mathcal{F}_{0}(\mathcal{X}):=\left\{\psi \in \mathcal{F}_{\mathrm{b}}(\mathcal{X}) \mid\right. & \text { there exists a number } n_{0} \text { such that } \\
& \left.\psi^{(n)}=0 \text { for all } n \geq n_{0}\right\}
\end{aligned}
$$

where $[X, Y]:=X Y-Y X$.

Let

$$
\phi(f):=\frac{a(f)+a(f)^{*}}{\sqrt{2}}, \quad f \in \mathcal{X},
$$

which is called the Segal field operator. It is shown that $\phi(f)$ is essentially self-adjoint on $\mathcal{F}_{0}(\mathcal{X})[16, \S \mathrm{X} .7]$. We denote its closure by the same symbol $\phi(f)$.

It follows from (2.3) that, for all $f, g \in L^{2}\left(\mathbb{R}^{d}\right)$,

$$
[\phi(f), \phi(g)]=i \Im\langle f, g\rangle
$$

on $\mathcal{F}_{0}(\mathcal{X})$. Moreover we have

$$
e^{i \phi(f)} e^{i \phi(g)}=e^{-i \Im\langle f, g\rangle} e^{i \phi(g)} e^{i \phi(f)}, \quad f, g \in \mathcal{X},
$$

which is called the Weyl relations of $\{\phi(f) \mid f \in \mathcal{X}\}[16, \S \mathrm{X} .7]$.

For every self-adjoint operator $S$ on $\mathcal{X}$, one can define a self-adjoint operator $d \Gamma(S)$, called the second quantization of $S([15, \mathrm{p} .302],[16, \S \mathrm{X} .7])$, by

$$
d \Gamma(S):=\oplus_{n=0}^{\infty} S^{(n)},
$$

with $S^{(0)}=0$ and $S^{(n)}$ is the closure of

$$
(\sum_{j=1}^{n} I \otimes \cdots \otimes \underbrace{j \text { th }}_{S} \otimes \cdots \otimes I) \mid \otimes_{\text {alg }}^{n} D(S),
$$


where $I$ denotes identity and $\otimes_{\text {alg }}^{n}$ algebraic tensor product. If $S$ is nonnegative, then so is $d \Gamma(S)$.

We assume that the Bose field is an $N$-component quantum field over $\mathbb{R}^{d}(d, N \in \mathbb{N})$. Hence the one-boson Hilbert space is taken to be

$$
\mathcal{W}:=\oplus^{N} L^{2}\left(\mathbb{R}^{d}\right)
$$

(the $N$ direct sum of $L^{2}\left(\mathbb{R}^{d}\right)$ ) and the Hilbert space of the Bose field is taken to be $\mathcal{F}_{\mathrm{b}}(\mathcal{W})$. Let $\omega$ be a Borel measurable function on $\mathbb{R}^{d}$ which is injective and $0<\omega(k)<\infty$ for a.e. $k \in \mathbb{R}^{d}$ with respect to (w.r.t.) the Lebesgue measure on $\mathbb{R}^{d}$. Then $\omega$ defines a multiplication operator on $\mathcal{W}$, which is nonnegative, injective and self-adjoint. We denote it by the same symbol $\omega\left(\omega f:=\left(\omega f_{1}, \cdots, \omega f_{N}\right), f=\left(f_{1}, \cdots, f_{N}\right) \in \mathcal{W}\right.$ with $\left.f_{i} \in D(\omega), i=1, \cdots, N\right)$. The function $\omega$ represents a dispersion relation of one free boson associated with the Bose field under consideration.

The free Hamiltonian of the Bose filed is defined by

$$
H_{\mathrm{b}}:=d \Gamma(\omega)
$$

acting on $\mathcal{F}_{\mathrm{b}}(\mathcal{W})$.

The Hilbert space of the coupled system of $\mathbf{S}$ and the Bose field is given by the tensor product

$$
\mathcal{F}:=\mathcal{H} \otimes \mathcal{F}_{\mathrm{b}}(\mathcal{W})
$$

Let $A$ be a self-adjoint operator on $\mathcal{H}$, which denotes physically the Hamiltonian of the quantum system $\mathbf{S}$ and $B_{j}(j=1, \cdots, J, J \in \mathbb{N})$ be a self-adjoint operator on $\mathcal{H}$ such that $\cap_{j=1}^{J} D\left(B_{j}\right)$ is dense in $\mathcal{H}$. Let $g_{j} \in \mathcal{W}, j=1, \cdots, J$. As a total Hamiltonian of the coupled system, we take the following operator:

$$
H(\lambda):=A \otimes I+I \otimes H_{\mathrm{b}}+\lambda \sum_{j=1}^{J} B_{j} \otimes \phi\left(g_{j}\right),
$$

where $\lambda \in \mathbb{R}$ is a constant parameter denoting the coupling constant of the system $\mathbf{S}$ and the Bose field system. The Hamiltonian $H(\lambda)$ gives a unification of Hamiltonians of some particle-field interaction models (cf. $[4,5])$.

In the previous papers $[4,5]$, the existence of a ground state of $H(\lambda)$ with $N=1$ is discussed under the assumption that $A$ has a ground state (hence $H(0)$ has a ground state). In the present paper, we consider the problem of enhanced binding on the model, i.e., the problem whether or not $H(\lambda)$ with $\lambda \neq 0$ has a ground state even if $A$ has no ground state(hence $H(0)$ has no ground state). We show that, under suitable hypotheses, the problem is solved affirmatively. For results on the problem of enhanced binding on the Pauli-Fierz model in non-relativistic QED, see $[8,9,10,11,14]$. The method taken in the present paper is similar to that used in [14], but we do not need such scalings as done in [14], at least on the level of a general theory.

We now formulate basic hypotheses. To do this, we first recall an important notion on commutativity of self-adjoint operators: 
Definition 2.1 We say that two self-adjoint operators $T$ and $S$ on a Hilbert space strongly commute (or $T$ strongly commutes with $S$ ) if their spectral meausres commute, i.e., for all Borel sets $I_{1}, I_{2} \subset \mathbb{R}, E_{T}\left(I_{1}\right) E_{S}\left(I_{2}\right)=E_{S}\left(I_{2}\right) E_{T}\left(I_{1}\right)$.

A family of self-adjoint operators $\left\{S_{j}\right\}_{j=1}^{n}$ on a Hilbert space is said to be strongly commuting if $S_{j}$ strongly commutes with $S_{l}$ for all $j, l=1, \cdots, n$ with $j \neq l$.

In what follows, we assume that $A$ is of the form

$$
A=A_{0}+A_{1}
$$

with $A_{0}$ a nonnegative self-adjoint operator and $A_{1}$ a symmetric operator.

Hypothesis I. $g_{j}, g_{j} / \omega^{3 / 2} \in \mathcal{W}(j=1, \cdots, J)$ and $\left\langle g_{j}(k), g_{l}(k)\right\rangle_{\mathbb{C}^{N}} \in \mathbb{R}$, a.e. $k, j, l=$ $1, \cdots, J$.

Hypothesis II. The operator $A_{1}$ is $A_{0}$-bounded, i.e., $D\left(A_{0}\right) \subset D\left(A_{1}\right)$ and there exist constants $a, b \geq 0$ such that, for all $u \in D\left(A_{0}\right)$,

$$
\left\|A_{1} u\right\| \leq a\left\|A_{0} u\right\|+b\|u\| .
$$

Hypothesis III. The operator $A_{0}$ strongly commutes with each $B_{j}(j=1, \cdots, J)$ and

$$
D\left(A_{0}\right) \subset \cap_{j, l=1}^{J} D\left(B_{j} B_{l}\right) .
$$

Moreover, there exist constants $c_{j}, d_{j} \geq 0$ such that, for all $u \in D\left(A_{0}^{1 / 2}\right)$,

$$
\left\|B_{j} u\right\| \leq c_{j}\left\|A_{0}^{1 / 2} u\right\|+d_{j}\|u\| \quad(j=1, \cdots, J) .
$$

Hypothesis IV. The set $\left\{B_{j}\right\}_{j=1}^{J}$ is a family of strongly commuting self-adjoint operators.

Hypothesis V. $D\left(A_{0}\right) \subset \cap_{j=1}^{J} D\left(B_{j} A_{1}\right) \cap D\left(A_{1} B_{j}\right)$ and $\left[B_{j}, A_{1}\right] \mid D\left(A_{0}\right)$ is bounded $(j=$ $1, \cdots, J)$. We denote the operator norm of $\left[B_{j}, A_{1}\right]$ by $\left\|\left[B_{j}, A_{1}\right]\right\|$.

We introduce an operator

$$
R_{B}:=\frac{1}{2} \sum_{j, l=1}^{J}\left\langle\frac{g_{j}}{\sqrt{\omega}}, \frac{g_{l}}{\sqrt{\omega}}\right\rangle_{\mathcal{W}} B_{j} B_{l} .
$$

and define

$$
A(\lambda):=A-\lambda^{2} R_{B}
$$

Under Hypotheses I-III, we have $D(A(\lambda))=D\left(A_{0}\right)$.

Let

$$
\Lambda:=\{\lambda \in \mathbb{R} \backslash\{0\} \mid A(\lambda) \text { is self-adjoint and bounded from below }\}
$$

Hypothesis VI. $\Lambda \neq \emptyset$. 
Remark 2.1 Assume Hypotheses I-III and suppose that

$$
a+\frac{\lambda^{2}}{2} \sum_{j, l=1}^{J}\left|\left\langle\frac{g_{j}}{\sqrt{\omega}}, \frac{g_{l}}{\sqrt{\omega}}\right\rangle\right| c_{j} c_{l}<1 .
$$

Then Hypothesis VI holds. This is proved as follows.

By Hypothesis III, we can show that

$$
\left\|B_{j} B_{l} u\right\| \leq c_{j} c_{l}\left\|A_{0} u\right\|+\left(c_{j} d_{l}+c_{l} d_{j}\right)\left\|A_{0}^{1 / 2} u\right\|+d_{j} d_{l}\|u\|, \quad u \in D\left(A_{0}\right) .
$$

Hence

$$
\begin{aligned}
\left\|\left(A_{1}-\lambda^{2} R_{B}\right) u\right\| \leq & \left(a+\frac{\lambda^{2}}{2} \sum_{j, l=1}^{J}\left|\left\langle\frac{g_{j}}{\sqrt{\omega}}, \frac{g_{l}}{\sqrt{\omega}}\right\rangle\right| c_{j} c_{l}\right)\left\|A_{0} u\right\| \\
& +\lambda^{2}\left(\sum_{j, l=1}^{J}\left|\left\langle\frac{g_{j}}{\sqrt{\omega}}, \frac{g_{l}}{\sqrt{\omega}}\right\rangle\right| c_{j} d_{l}\right)\left\|A_{0}^{1 / 2} u\right\| \\
& +\left(b+\frac{\lambda^{2}}{2} \sum_{j, l=1}^{J}\left|\left\langle\frac{g_{j}}{\sqrt{\omega}}, \frac{g_{l}}{\sqrt{\omega}}\right\rangle\right| d_{j} d_{l}\right)\|u\| .
\end{aligned}
$$

Since $A_{0}^{1 / 2}$ is infinitesimally small w.r.t. $A_{0}$, it follows under condition (2.20) that $A_{1}-$ $\lambda^{2} R_{B}$ is relatively bounded w.r.t. $A_{0}$ with relative bound small than 1 . Hence, by the Kato-Rellich theorem, $A(\lambda)=A_{0}+A_{1}-\lambda^{2} R_{B}$ is self-adjoint with $D(A(\lambda))=D\left(A_{0}\right)$ and bounded from below.

A result on the self-adjointness of $H(\lambda)$ is given by the following theorem.

Theorem 2.2 Assume Hypotheses $I-V I$. Then for all $\lambda \in \Lambda, H(\lambda)$ is self-adjoint with $D(H(\lambda))=D\left(A_{0} \otimes I\right) \cap D\left(I \otimes H_{\mathrm{b}}\right)$ and bounded from below.

This theorem is proved in Section 3.

To establish an existence theorem of a ground state of $H(\lambda)$ without the assumption that $A$ has a ground state, we need additional conditions.

Hypothesis VII. The function $\omega$ is continuous on $\mathbb{R}^{d}$ with

$$
\lim _{|k| \rightarrow \infty} \omega(k)=\infty
$$

and there exist constants $\gamma>0$ and $C>0$ such that

$$
\left|\omega(k)-\omega\left(k^{\prime}\right)\right| \leq C\left|k-k^{\prime}\right|^{\gamma}\left(1+\omega(k)+\omega\left(k^{\prime}\right)\right), \quad k, k^{\prime} \in \mathbf{R}^{d} .
$$

In general the existence of a ground state of $H(\lambda)$ may depend on whether

$$
m:=\text { ess. } \inf _{k \in \mathbb{R}^{d}} \omega(k)
$$


is positive or zero [6], where ess. inf means essential infimum. We say that the Bose field under the consideration is massive (resp. massless) if $m>0$ (resp. $m=0$ ). We first establish a theorem on the existence of a ground state of $H(\lambda)$ in the massive case.

For $s \geq 0$, we introduce a constant $c_{s}(g)$ by

$$
c_{s}(g):=\sqrt{2} \sum_{j=1}^{J}\left\|\left[B_{j}, A_{1}\right]\right\|\left\|\frac{g_{j}}{\omega^{s}}\right\|
$$

provided that $g_{j} / \omega^{s} \in \mathcal{W}$. We set

$$
\Sigma_{\lambda}:=\inf \sigma_{\mathrm{ess}}(A(\lambda))
$$

Remark 2.2 If $m>0$, then the condition $g_{j} \in \mathcal{W}$ implies that $g_{j} / \omega^{s} \in \mathcal{W}$ for all $s>0$. Hence, in this case, Hypothesis I is replaced by the condition that $g_{j} \in \mathcal{W}(j=1, \cdots, J)$ and $\left\langle g_{j}(k), g_{l}(k)\right\rangle_{\mathbb{C}^{N}} \in \mathbb{R}$, a.e. $k, j, l=1, \cdots, J$.

Theorem 2.3 (Enhanced binding in the massive case). Consider the case $m>0$. Assume Hypotheses I-VII. Suppose that $\lambda \in \Lambda$ and

$$
\Sigma_{\lambda}-E_{0}(A(\lambda))>m+\frac{1}{2} \lambda^{2} c_{3 / 2}(g)^{2}+|\lambda| c_{1}(g) .
$$

Then $H(\lambda)$ has purely discrete spectrum in the interval $\left[E_{0}(H(\lambda)), E_{0}(H(\lambda))+m\right)$. In particular, $H(\lambda)$ has has a ground state.

Remark 2.3 Condition (2.25) implies that $E_{0}(A(\lambda))$ is a discrete eigenvalue of $A(\lambda)$ and hence $A(\lambda)$ has a finite number of ground states.

A new point of Theorem 2.3 is in that the existence of a ground state of $A$ is not assumed.. Theorem 2.3 is proved in Section 4.

Theorem 2.4 (Enhanced binding in the massless case). Consider the case $m=0$. Assume Hypotheses $I-V I I$ with $g_{j} / \omega^{2} \in \mathcal{W}(j=1, \cdots, J)$ in addition. Suppose that

$$
\Sigma_{\lambda}-E_{0}(A(\lambda))>\frac{1}{2} \lambda^{2} c_{3 / 2}(g)^{2}+|\lambda| c_{1}(g) .
$$

and

$$
\frac{\lambda^{2} c_{1}(g)^{2}}{\left[\Sigma_{\lambda}-E_{0}(H(\lambda))\right]^{2}}+\left\{\frac{2 \lambda^{2} c_{1}(g)^{2}}{\left[\Sigma_{\lambda}-E_{0}(H(\lambda))\right]^{2}}+1\right\} \frac{\lambda^{2}}{2} c_{2}(g)^{2}<1 .
$$

Then $H(\lambda)$ has a ground state.

We prove this theorem in Section 5. 


\section{Self-adjointness of the Total Hamiltonian}

Generally speaking, in considering the enhanced binding problem of a quantum field model, it would be desirable to establish the self-adjointness of the Hamiltonian of the model for a wider range of the coupling constant. For this purpose the method used in [4], which employs the Kato-Rellich theorem, is not useful. Here we take another approach which is used in [1]: we prove Theorem 2.2 by making a suitable unitary transformation of $H(\lambda)$.

We need some lemmas.

Lemma 3.1 Let $\mathcal{X}$ and $\mathcal{Y}$ be Hilbert spaces. Let $\left\{X_{j}\right\}_{j=1}^{J}$ (resp. $\left\{Y_{j}\right\}_{j=1}^{J}$ ) be a family of strongly commuting self-adjoint adjoint operators on $\mathcal{X}$ (resp. $\mathcal{Y})$. Then $\left\{X_{j} \otimes Y_{j}\right\}_{j=1}^{J}$ is a family of strongly commuting self-adjoint operators on $\mathcal{X} \otimes \mathcal{Y}$. Moreover, if $W$ (resp. $U$ ) is a self-adjoint operator on $\mathcal{X}$ (resp. $\mathcal{Y}$ ) strongly commuting with each $X_{j}$ (resp. $Y_{j}$ ), then $W \otimes I$ (resp. $I \otimes U$ ) strongly commutes with each $X_{j} \otimes Y_{j}$.

Proof. It is a well known fact that each $X_{j} \otimes Y_{j}$ is self-adjoint on $\mathcal{X} \otimes \mathcal{Y}$ (e.g., [15, $\S$ VIII.10]). Moreover there exists a two-dimensional spectral measure $E_{j}$ such that, for all Borel sets $I_{1}, I_{2} \subset \mathbb{R}, E_{j}\left(I_{1} \times I_{2}\right)=E_{X_{j}}\left(I_{1}\right) \otimes E_{Y_{j}}\left(I_{2}\right)$ and $X_{j} \otimes I=\int_{\mathbb{R}^{2}} x d E_{j}(x, y), Y_{j}=$ $\int_{\mathbb{R}^{2}} y d E_{j}(x, y), f\left(X_{j} \otimes Y_{j}\right)=\int_{\mathbb{R}^{2}} f(x y) d E_{j}(x, y)$. It follows that, for all Borel sets $K \subset \mathbb{R}$, $E_{X_{j} \otimes Y_{j}}(K)=E_{j}\left(\left\{(x, y) \in \mathbb{R}^{2} \mid x y \in K\right\}\right)$. On the other hand, the strong commutativity of $X_{j}$ 's and that of $Y_{j}$ 's imply that $\left\{E_{j}(\cdot)\right\}_{j=1}^{J}$ is a family of commuting orthogonal projections. Hence, for all Borel sets $I_{1}, I_{2} \subset \mathbb{R}$ and $j, l=1, \cdots, J, E_{X_{j} \otimes Y_{j}}\left(I_{1}\right)$ commutes with $E_{X_{l} \otimes Y_{l}}\left(I_{2}\right)$.

Let $W$ and $U$ be as above. Then $E_{W}\left(I_{1}\right) E_{X_{j}}\left(I_{2}\right)=E_{X_{j}}\left(I_{2}\right) E_{W}\left(I_{1}\right)$, which implies the commutativity of $E_{W \otimes I}$ and $E_{j}$. Hence $E_{W \otimes I}$ commutes with $E_{X_{j} \otimes Y_{j}}$. Thus $W \otimes I$ strongly commutes with $X_{j} \otimes Y_{j}$. Similarly one can show that $I \otimes U$ strongly commutes with $X_{j} \otimes Y_{j}$.

The following fact is well known or easy to prove (e.g., [3, Lemma 2.33]).

Lemma 3.2 Let $\left\{S_{j}\right\}_{j=1}^{J}$ be a family of strongly commuting self-adjoint operators on a Hilbert space. Then $S:=\sum_{j=1}^{J} S_{j}$ is essentially self-adjoint and, for all $t \in \mathbb{R}$,

$$
e^{i t \bar{S}}=\prod_{j=1}^{J} e^{i t S_{j}},
$$

where $\bar{S}$ denotes the closure of $S$ and the order of the factors on the right hand side (r.h.s.) is arbitrary.

Lemma 3.3 Let $\mathcal{X}$ be a separable complex Hilbert space and $h_{j} \in \mathcal{X}(j=1, \cdots, J)$ such that $\left\langle h_{j}, h_{l}\right\rangle_{\mathcal{X}} \in \mathbb{R}, j, l=1, \cdots, J$. Then $\left\{\phi\left(h_{j}\right)\right\}_{j=1}^{J}$ is a family of strongly ommuting self-adjoint operators on $\mathcal{F}_{\mathrm{b}}(\mathcal{X})$.

Proof. By the present assumption, $\Im\left\langle h_{j}, h_{l}\right\rangle=0$. Hence, by the Weyl relations (2.7), $e^{i t \phi\left(h_{j}\right)}$ commutes with $e^{i s \phi\left(h_{l}\right)}$ for all $s, t \in \mathbb{R}$ and $j, l=1, \cdots, J$. Hence, by a general criterion [15, Theorem VIII.13], $\phi\left(h_{j}\right)$ strongly commutes with $\phi\left(h_{l}\right)(j, l=1, \cdots, J)$. 
Let

$$
T_{j}:=B_{j} \otimes \phi\left(i \frac{g_{j}}{\omega}\right)
$$

Then the operator

$$
T:=\sum_{j=1}^{J} T_{j}
$$

is a symmetric operator with $D(T) \supset \cap_{j=1}^{J} D\left(B_{j}\right) \otimes_{\text {alg }} \mathcal{F}_{0}(\mathcal{W})$. We denote the closure of $T$ by the same symbol $T$.

Lemma 3.4 Assume Hypotheses I and IV. Then:

(i) $\left\{T_{j}\right\}_{j=1}^{J}$ is a family of strongly commuting self-adjoint operators.

(ii) $T$ is essentially self-adjoint on $\cap_{j=1}^{J} D\left(T_{j}\right)$ and, for all $s \in \mathbb{R}$,

$$
e^{i s T}=\prod_{j=1}^{J} e^{i s T_{j}},
$$

where the order of the factors on the r.h.s. is arbitrary.

Proof. (i) By Lemmas 3.1, 3.3 and Hypothesis IV, $\left\{T_{j}\right\}_{j=1}^{J}$ is a family of strongly commuting self-adjoint operators.

(ii) By part (i) and Lemma 3.2, $T$ is essentially self-adjoint and (3.4) holds.

Lemma 3.5 Assume Hypotheses I, III and IV. Then T strongly commutes with $A_{0} \otimes I$.

Proof. By Lemma 3.1 and Hypothesis III, $A_{0} \otimes I$ strongly commutes with each $T_{j}$, which implies that, for all $s, t \in \mathbb{R}, e^{i t A_{0} \otimes I} e^{i s T_{j}}=e^{i s T_{j}} e^{i t A_{0} \otimes I}$. By this equation and (3.4), $e^{i t A_{0} \otimes I} e^{i s T}=e^{i s T} e^{i t A_{0} \otimes I}$. Hence, by a general criterion [15, Theorem VIII.13], $T$ strongly commutes with $A_{0} \otimes I$.

The following fact is well known (e.g., [3, p.516, Lemma 12-5]).

Lemma 3.6 Let $\mathcal{X}$ be a Hilbert space and $S$ be a nonnegative, injective self-adjoint operator on $\mathcal{X}$. Let $g \in D(S)$. Then

$$
e^{i \phi(i g)} D(d \Gamma(S))=D(d \Gamma(S))
$$

and

$$
e^{i \phi(i g)} d \Gamma(S) e^{-i \phi(i g)}=d \Gamma(S)+\phi(S g)+\frac{1}{2}\langle g, S g\rangle
$$

Suppose that Hypotheses I and IV hold. Then, by Lemma 3.4, we can define a unitary operator

$$
U(\lambda):=e^{-i \lambda T}
$$

We set

$$
L:=A_{0} \otimes I+I \otimes H_{\mathrm{b}}
$$


which is nonnegative.

We introduce an operator

$$
\widetilde{H}(\lambda):=A(\lambda) \otimes I+I \otimes H_{\mathrm{b}}+\delta A_{1}(\lambda),
$$

where

$$
\delta A_{1}(\lambda):=U(\lambda)\left(A_{1} \otimes I\right) U(\lambda)^{-1}-A_{1} \otimes I
$$

Lemma 3.7 Assume Hypotheses $I-I V$. Then, for all $\lambda \in \mathbb{R}$,

$$
U(\lambda) D(L)=D(L)
$$

and, for all $\Psi \in D(L)$,

$$
U(\lambda) H(\lambda) U(\lambda)^{-1}=\widetilde{H}(\lambda) \Psi
$$

Proof. By Hypothesis IV, there exists a $J$-dimensional spectral measure $E$ such that, for all Borel sets $I_{j} \subset \mathbb{R}(j=1, \cdots, J), E\left(I_{1} \times \cdots \times I_{J}\right)=E_{B_{1}}\left(I_{1}\right) \cdots E_{B_{J}}\left(I_{J}\right)$ and $B_{j}=\int_{\mathbb{R}^{J}} \xi_{j} d E(\xi)\left(\xi=\left(\xi_{1}, \cdots, \xi_{J}\right) \in \mathbb{R}^{J}\right)$. Let $u, v \in D\left(A_{0}\right)$ and $\psi, \varphi \in D\left(H_{\mathrm{b}}\right)$. Set $\Psi=u \otimes \psi$ and $\Phi=v \otimes \varphi$. Then $\Psi, \Phi \in D(L)$ and

$$
\left\langle I \otimes H_{b} \Psi, U(\lambda) \Phi\right\rangle=\int_{\mathbb{R}^{J}}\left\langle H_{\mathrm{b}} \psi, e^{-i \phi\left(i G_{\xi}\right)} \varphi\right\rangle d\langle u, E(\xi) v\rangle,
$$

where $G_{\xi}=\lambda \sum_{j=1}^{J} \xi_{j} g_{j} / \omega \in \mathcal{W}$. By Lemma 3.6, we have

$$
\left\langle H_{\mathrm{b}} \psi, e^{-i \phi\left(i G_{\xi}\right)} \varphi\right\rangle=\left\langle\psi, e^{-i \phi\left(i G_{\xi}\right)}\left(H_{\mathrm{b}}+\phi\left(\omega G_{\xi}\right)+\frac{1}{2}\left\langle G_{\xi}, \omega G_{\xi}\right\rangle\right) \varphi\right\rangle .
$$

Hence

$$
\left\langle I \otimes H_{b} \Psi, U(\lambda) \Phi\right\rangle=\left\langle\Psi, U(\lambda)\left(I \otimes H_{\mathrm{b}}+\lambda \sum_{j=1}^{J} B_{j} \otimes \phi\left(g_{j}\right)+\lambda^{2} R_{B} \otimes I\right) \Phi\right\rangle .
$$

This extends to all $\Psi, \Phi \in D\left(A_{0}\right) \otimes_{\text {alg }} D\left(H_{\mathrm{b}}\right)$. Using the well known estimates

$$
\begin{aligned}
& \|a(f) \psi\| \leq\left\|\frac{f}{\sqrt{\omega}}\right\|\left\|H_{\mathrm{b}}^{1 / 2} \psi\right\|, \\
& \left\|a(f)^{*} \psi\right\| \leq\left\|\frac{f}{\sqrt{\omega}}\right\|\left\|H_{\mathrm{b}}^{1 / 2} \psi\right\|+\|f\|\|\psi\|, \quad \psi \in D\left(H_{\mathrm{b}}^{1 / 2}\right), f \in D\left(\omega^{-1 / 2}\right),
\end{aligned}
$$

and Hypothesis III, we can show that

$$
\left\|B_{j} \otimes \phi\left(g_{j}\right) \Psi\right\| \leq C\|(L+1) \Psi\|, \quad \Psi \in D(L),
$$

where $C>0$ is a constant. By $(2.21), R_{B}$ is $A_{0}$-bounded. Hence

$$
\left\|\left(I \otimes H_{\mathrm{b}}+\lambda \sum_{j=1}^{J} B_{j} \otimes \phi\left(g_{j}\right)+\lambda^{2} R_{B} \otimes I\right) \Xi\right\| \leq C^{\prime}\|(L+1) \Xi\|, \quad \Xi \in D(L),
$$


where $C^{\prime}>0$ is a constant. By this estimate and the fact that $D\left(A_{0}\right) \otimes_{\mathrm{alg}} D\left(H_{\mathrm{b}}\right)$ is a core for $L$, (3.13) extends (via a limiting argument) to all $\Phi \in D(L)$. Moreover, $D\left(A_{0}\right) \otimes_{\text {alg }} D\left(H_{\mathrm{b}}\right)$ is a core for $I \otimes H_{\mathrm{b}},(3.13)$ extends also to all $\Psi \in D\left(I \otimes H_{\mathrm{b}}\right)$. Thus, for all $\Phi \in D(L)$, the vector $U(\lambda) \Phi$ is in $D\left(I \otimes H_{\mathrm{b}}\right)$ and

$$
I \otimes H_{\mathrm{b}} U(\lambda) \Phi=U(\lambda)\left(I \otimes H_{\mathrm{b}}+\lambda \sum_{j=1}^{J} B_{j} \otimes \phi\left(g_{j}\right)+\lambda^{2} R_{B} \otimes I\right) \Phi .
$$

The strong commutativity of $A_{0} \otimes I$ and $T$ (Lemma 3.5) implies that $U(\lambda) D\left(A_{0} \otimes I\right) \subset$ $D\left(A_{0} \otimes I\right)$. Thus $U(\lambda) D(L) \subset D(L)$. Since $U(\lambda)$ is unitary, it follows that $D(L) \subset$ $U(\lambda)^{-1} D(L)=U(-\lambda) D(L)$. Since $\lambda \in \mathbb{R}$ is arbitrary, we obtain (3.11). Eq.(3.18) implies (3.12).

In view of Lemma 3.7, we first prove the self-adjointness of $\widetilde{H}(\lambda)$ (Theorem 3.11 below).

We denote by $\overline{\left[B_{j}, A_{1}\right]}$ the closure of $\left[B_{j}, A_{1}\right] \mid D\left(A_{0}\right)$ which, by Hypothesis $\mathrm{V}$, is bounded with $D\left(\overline{\left[B_{j}, A_{1}\right]}\right)=\mathcal{H}$.

Lemma 3.8 Assume Hypotheses $I$ and $V$. Then $\left.D\left(I \otimes H_{\mathrm{b}}^{1 / 2}\right)\right) \subset D\left(\overline{\left[B_{j}, A_{1}\right]} \otimes \phi\left(i g_{j} / \omega\right)\right)$ and, for all $\Psi \in D\left(I \otimes H_{\mathrm{b}}^{1 / 2}\right)$,

$$
\left\|\overline{\left[B_{j}, A_{1}\right]} \otimes \phi\left(i g_{j} / \omega\right) \Psi\right\| \leq\left\|\left[B_{j}, A_{1}\right]\right\|\left(\sqrt{2}\left\|\frac{g_{j}}{\omega^{3 / 2}}\right\|\left\|I \otimes H_{\mathrm{b}}^{1 / 2} \Psi\right\|+\frac{1}{\sqrt{2}}\left\|\frac{g_{j}}{\omega}\right\|\|\Psi\|\right) .
$$

Proof. By (3.14) and (3.15), we have

$$
\|\phi(f) \psi\| \leq \sqrt{2}\left\|\frac{f}{\sqrt{\omega}}\right\|\left\|H_{\mathrm{b}}^{1 / 2} \psi\right\|+\frac{1}{\sqrt{2}}\|f\|\|\psi\|, \quad f \in D\left(\omega^{-1 / 2}\right), \psi \in D\left(H_{\mathrm{b}}^{1 / 2}\right) .
$$

Hence, for all $\Psi \in D\left(A_{0}\right) \otimes_{\text {alg }} D\left(H_{\mathrm{b}}^{1 / 2}\right)$,

$$
\begin{aligned}
\left\|\overline{\left[B_{j}, A_{1}\right]} \otimes \phi\left(i g_{j} / \omega\right) \Psi\right\| & \leq\left\|\left[B_{j}, A_{1}\right]\right\|\left\|I \otimes \phi\left(i g_{j} / \omega\right) \Psi\right\| \\
& \leq\left\|\left[B_{j}, A_{1}\right]\right\|\left(\sqrt{2}\left\|g_{j} / \omega^{3 / 2}\right\|\left\|I \otimes H_{\mathrm{b}}^{1 / 2} \Psi\right\|+\left\|g_{j} / \omega\right\|\|\Psi\| / \sqrt{2}\right) .
\end{aligned}
$$

Hence (3.19) holds for all $\Psi \in D\left(A_{0}\right) \otimes_{\mathrm{alg}} D\left(H_{\mathrm{b}}^{1 / 2}\right)$. Since $D\left(A_{0}\right) \otimes_{\mathrm{alg}} D\left(H_{\mathrm{b}}^{1 / 2}\right)$ is a core for $I \otimes H_{\mathrm{b}}^{1 / 2},(3.19)$ extends to all $\Psi \in D\left(I \otimes H_{\mathrm{b}}^{1 / 2}\right)$ showing $\left.D\left(I \otimes H_{\mathrm{b}}^{1 / 2}\right)\right) \subset$ $D\left(\overline{\left[B_{j}, A_{1}\right]} \otimes \phi\left(i g_{j} / \omega\right)\right)$ as well.

We set

$$
Y:=\sum_{j=1}^{J} \overline{\left[B_{j}, A_{1}\right]} \otimes \phi\left(i \frac{g_{j}}{\omega}\right)
$$

By Lemma 3.8, we have

$$
D\left(I \otimes H_{\mathrm{b}}\right) \subset D(Y)
$$

Lemma 3.9 Assume Hypotheses I-III and Hypothesis V. Then, for all $\Psi, \Phi \in D(L)$

$$
\left\langle T \Psi, A_{1} \otimes I \Phi\right\rangle-\left\langle A_{1} \otimes I \Psi, T \Phi\right\rangle=\langle\Psi, Y \Phi\rangle .
$$


Proof. It is easy to see that, for all $\Psi, \Phi \in D\left(A_{0}\right) \otimes_{\text {alg }} D\left(H_{\mathrm{b}}\right),(3.22)$ holds. By (3.16), $T$ is $L$-bounded. By Hypothesis II, one can show that $A_{1} \otimes I$ is $L$-boounded. By Lemma 3.8, $Y$ also is $L$-bounded. Since $D\left(A_{0}\right) \otimes_{\text {alg }} D\left(H_{\mathrm{b}}\right)$ is a core for $L,(3.22)$ extends to all $\Psi, \Phi \in D(L)$.

Lemma 3.10 Assume Hypotheses $I-V$. Then, $D(L) \subset D\left(\delta A_{1}(\lambda)\right)$ and, for all $\Psi \in D(L)$,

$$
\left\|\delta A_{1}(\lambda) \Psi\right\| \leq|\lambda|\left(c_{3 / 2}(g)\left\|I \otimes H_{\mathrm{b}}^{1 / 2} \Psi\right\|+\frac{1}{2} c_{1}(g)\|\Psi\|\right),
$$

where $c_{s}(g)$ is defined by (2.23).

Proof. Let $A_{1}(\lambda)=U(\lambda) A_{1} \otimes I U(\lambda)^{-1}$ and $\Psi, \Phi \in D(L)$. Then, applying Proposition A.1 in Appendix A with $H=-\lambda T, S=A_{1} \otimes I$ and $K=A_{0} \otimes I$, we see that the function: $t \mapsto\left\langle\Phi, A_{1}(t \lambda) \Psi\right\rangle(t \in \mathbb{R})$ is differentiable and

$$
\begin{aligned}
\frac{d}{d t}\left\langle\Phi, A_{1}(t \lambda) \Psi\right\rangle= & -i \lambda\left\{\left\langle T U(t \lambda)^{-1} \Phi, A_{1} \otimes I U(t \lambda)^{-1} \Psi\right\rangle\right. \\
& \left.-\left\langle A_{1} \otimes I U(t \lambda)^{-1} \Phi, T U(t \lambda)^{-1} \Psi\right\rangle\right\},
\end{aligned}
$$

which, together with Lemma 3.9, yields that

$$
\frac{d}{d t}\left\langle\Phi, A_{1}(t \lambda) \Psi\right\rangle=-i \lambda\left\langle U(t \lambda)^{-1} \Phi, Y U(t \lambda)^{-1} \Psi\right\rangle
$$

Integrating this equation from $t=0$ to $t=1$, we obtain

$$
\left\langle\Phi, \delta A_{1}(\lambda) \Psi\right\rangle=-i \lambda \int_{0}^{1}\left\langle U(t \lambda)^{-1} \Phi, Y U(t \lambda)^{-1} \Psi\right\rangle d t
$$

Hence

$$
\left|\left\langle\Phi, \delta A_{1}(\lambda) \Psi\right\rangle\right| \leq|\lambda| \int_{0}^{1}\|\Phi\|\left\|Y U(t \lambda)^{-1} \Psi\right\| d t
$$

which implies that

$$
\left\|\delta A_{1}(\lambda) \Psi\right\| \leq|\lambda| \int_{0}^{1}\left\|Y U(t \lambda)^{-1} \Psi\right\| d t
$$

We have

$$
\begin{aligned}
\left\|Y U(t \lambda)^{-1} \Psi\right\| & \leq \sum_{j=1}^{J}\left\|\left[B_{j}, A_{1}\right]\right\|\left\|I \otimes \phi\left(i g_{j} / \omega\right) U(t \lambda)^{-1} \Psi\right\| \\
& =\sum_{j=1}^{J}\left\|\left[B_{j}, A_{1}\right]\right\|\left\|I \otimes \phi\left(i g_{j} / \omega\right) \Psi\right\|,
\end{aligned}
$$

where we have used the strong commutativity of $I \otimes \phi\left(i g_{j} / \omega\right)$ and $U(t \lambda)^{-1}$. Hence

$$
\left\|\delta A_{1}(\lambda) \Psi\right\| \leq|\lambda| \sum_{j=1}^{J}\left\|\left[B_{j}, A_{1}\right]\right\|\left\|I \otimes \phi\left(i g_{j} / \omega\right) \Psi\right\| .
$$

Using this estimate and (3.20), we obtain (3.23).

Let

$$
L(\lambda):=A(\lambda) \otimes I+I \otimes H_{\mathrm{b}}
$$


Theorem 3.11 Assume Hypotheses I-VI. Then, for all $\lambda \in \Lambda, \widetilde{H}(\lambda)$ is self-adjoint with $D(\widetilde{H}(\lambda))=D(L)$ and bounded from below. Moreover, every core of $L(\lambda)$ is a core of $\widetilde{H}(\lambda)$.

Proof. We can write

$$
\widetilde{H}(\lambda)=L(\lambda)+\delta A_{1}(\lambda)
$$

Let $\lambda \in \Lambda$. Then, by the definition of $\Lambda, L(\lambda)$ is self-adjoint and bounded from below. It is easy to see that $I \otimes H_{\mathrm{b}}^{1 / 2}$ is infinitesimally small with respect to (w.r.t.) $I \otimes H_{\mathrm{b}}$. Since $A(\lambda) \otimes I$ is bounded from below, it follows that $I \otimes H_{\mathrm{b}}^{1 / 2}$ is infinitesimally small with respect to (w.r.t.) $L(\lambda)$. Therefore, by Lemma 3.10, $\delta A_{1}(\lambda)$ is infinitesimally small w.r.t. $L(\lambda)$. Thus, by the Kato-Rellich theorem (e.g., [16, p.162, Theorem X.12] ), $\widetilde{H}(\lambda)$ is self-adjoint on $D(L(\lambda))=D\left(A_{0} \otimes I\right) \cap D\left(I \otimes H_{\mathrm{b}}\right)$, bounded from below and every core of $L(\lambda)$ is a core of $\widetilde{H}(\lambda)$.

Proof of Theorem 2.2

We have $D(L(\lambda))=D(L)$. By Lemma 3.7 and Theorem 3.11, $H(\lambda)$ is self-adjoint on $D(L)$ and bounded from below.

Corollary 3.12 Assume Hypotheses I-VI. Then, for all $\lambda \in \Lambda$,

$$
E_{0}(A(\lambda))-\frac{\lambda^{2}}{4} c_{3 / 2}(g)^{2}-\frac{|\lambda|}{2} c_{1}(g) \leq E_{0}(H(\lambda)) \leq E_{0}(A(\lambda))+\frac{1}{2}|\lambda| c_{1}(g) .
$$

Proof. By Lemma 3.7 and Theorem 2.2, we have

$$
E_{0}(H(\lambda))=E_{0}(\widetilde{H}(\lambda)) .
$$

Hence we need only to prove $(3.27)$ with $E_{0}(H(\lambda))$ replaced by $E_{0}(\widetilde{H}(\lambda))$.

Let $\Psi \in D(L)$ with $\|\Psi\|=1$. Then, using (3.23), we have

$$
\begin{aligned}
\langle\Psi, \widetilde{H}(\lambda) \Psi\rangle & \geq\langle\Psi, A(\lambda) \otimes I \Psi\rangle+\left\langle\Psi, I \otimes H_{\mathrm{b}} \Psi\right\rangle-\left\|\delta A_{1}(\lambda) \Psi\right\| \\
& \geq E_{0}(A(\lambda))+\left\|I \otimes H_{\mathrm{b}}^{1 / 2} \Psi\right\|^{2}-|\lambda| c_{3 / 2}(g)\left\|I \otimes H_{\mathrm{b}}^{1 / 2} \Psi\right\|-\frac{|\lambda|}{2} c_{1}(g) \\
& \geq E_{0}(A(\lambda))-\frac{\lambda^{2} c_{3 / 2}(g)^{2}}{4}-\frac{|\lambda|}{2} c_{1}(g),
\end{aligned}
$$

which, combined with the variational principle, yields the first inequality in (3.27).

Let $\Omega \in \mathcal{F}_{\mathrm{b}}(\mathcal{W})$ be the Fock vacuum: $\Omega^{(0)}=1, \Omega^{(n)}=0, n \geq 1$. Then we have

$$
H_{\mathrm{b}} \Omega=0 .
$$

Hence, for all $u \in D\left(A_{0}\right)$ with $\|u\|=1$, we have by the variational principle

$$
E_{0}(\widetilde{H}(\lambda)) \leq\langle u, A(\lambda) u\rangle+\left\langle u \otimes \Omega, \delta A_{1}(\lambda) u \otimes \Omega\right\rangle .
$$

By (3.23) and the Schwarz inequality, we have

$$
\left\langle u \otimes \Omega, \delta A_{1}(\lambda) u \otimes \Omega\right\rangle \leq \frac{1}{2}|\lambda| c_{1}(g) .
$$


Hence

$$
E_{0}(\widetilde{H}(\lambda)) \leq\langle u, A(\lambda) u\rangle+\frac{1}{2}|\lambda| c_{1}(g) .
$$

Applying the variational principle again, we obtain (3.27) with $E_{0}(H(\lambda))$ replaced by $E_{0}(\widetilde{H}(\lambda))$.

\section{Existence of a Ground State in the Massive Case}

In the present case, methods used in $[4,5]$ is not applied directly to proving Theorem 2.3, because the existence of a ground state of $A$ is not assumed. Thus we need a new idea. We note Lemma 3.7, which tells us that $H(\lambda)$ has a ground state if and only if $\widetilde{H}(\lambda)$ does. Hence one may prove the existence of a ground state of $H(\lambda)$ by proving that of $\widetilde{H}(\lambda)$. We use this structure.

Throughout this section we assume Hypothesis I-VII.

For a parameter $V>0$, we define a lattice

$$
\Gamma_{V}:=\frac{2 \pi \mathbb{Z}^{d}}{V}=\left\{k=\left(k_{1}, \cdots, k_{d}\right) \mid k_{j}=\frac{2 \pi n_{j}}{V}, n_{j} \in \mathbb{Z}, j=1, \cdots, d\right\} .
$$

We denote by $\ell^{2}\left(\Gamma_{V}\right)$ the Hilbert space of square sumable sequences indexed by $\Gamma_{V}$ :

$$
\ell^{2}\left(\Gamma_{V}\right):=\left\{f:\left.\Gamma_{V} \rightarrow \mathbb{C}\left|\sum_{k \in \Gamma_{V}}\right| f(k)\right|^{2}<\infty\right\} .
$$

Each element $f$ in $\ell^{2}\left(\Gamma_{V}\right)$ can be identified with a piecewise constant function in $L^{2}\left(\mathbb{R}^{d}\right)$ which is a constant on each cube

$$
C(k, V):=\left[k_{1}-\frac{\pi}{V}, k_{1}+\frac{\pi}{V}\right) \times \cdots \times\left[k_{d}-\frac{\pi}{V}, k_{d}+\frac{\pi}{V}\right) \subset \mathbb{R}^{d}
$$

centered about a lattice point $k \in \Gamma_{V}$. In this identification, $\ell^{2}\left(\Gamma_{V}\right)$ is a closed subspace of $L^{2}\left(\mathbb{R}^{d}\right)$. Then, putting

$$
\mathcal{W}_{V}:=\oplus^{N} \ell^{2}\left(\Gamma_{V}\right)
$$

we have a natural orthogonal decomposition

$$
\mathcal{W}=\mathcal{W}_{V} \oplus \mathcal{W}_{V}^{\perp}
$$

Hence

$$
\mathcal{F}_{\mathrm{b}}(\mathcal{W})=\mathcal{F}_{\mathrm{b}}\left(\mathcal{W}_{V}\right) \otimes \mathcal{F}_{\mathrm{b}}\left(\mathcal{W}_{V}^{\perp}\right)
$$

We define

$$
\omega_{V}(k)=\omega\left(k_{V}\right), \quad k \in \mathbb{R}^{d}
$$

with $k_{V}$ a lattice point closed to $k$ :

$$
k_{V} \in \Gamma_{V}, \quad\left|k_{j}-\left(k_{V}\right)_{j}\right| \leq \frac{\pi}{V}, \quad j=1, \cdots, d, \quad k \in C\left(k_{V}, V\right) .
$$


Let

$$
H_{\mathrm{b}, V}:=d \Gamma\left(\omega_{V}\right)
$$

the second quantization of $\omega_{V}$.

For technical reasons, we assume the following as a preliminary hypothesis:

Hypothesis VIII. Each $g_{j}: \mathbb{R}^{d} \rightarrow \mathbb{C}^{N}$ is continuous with $g_{j}, g_{j} / \omega^{3 / 2} \in \mathcal{W}$ and $\left\langle g_{j}(k), g_{l}(k)\right\rangle_{\mathbb{C}^{N}} \in \mathbb{R}, j, l=1, \cdots, J, k \in \mathbb{R}^{d}$.

Let $C, \gamma$ be the constants in Hypothesis VII. In what follows we assume that $m>0$ $(m$ is defined by $(2.22)$ ) and

$$
C_{V}:=C d^{\gamma / 2}\left(\frac{\pi}{V}\right)^{\gamma}\left(1+\frac{1}{2 m}\right)<1 .
$$

Condition (4.3) is equivalent to $V>V_{0}$, where $V_{0}$ is the constant defined by

$$
C_{V_{0}}=1 .
$$

For a constant $K>0$, we define a function $g_{j, K, V}: \mathbb{R}^{d} \rightarrow \mathbb{C}^{N}(j=1, \cdots, J)$ by

$$
g_{j, K, V}(k):=\sum_{\ell \in \Gamma_{V},\left|\ell_{i}\right| \leq K, i=1, \cdots, d} g_{j}(\ell) \chi_{C(\ell, V)}(k), k \in \mathbb{R}^{d}
$$

where $\chi_{S}$ denotes the characteristic fucntion of the set $S$. We introduce a lattice approximation version for $H(\lambda)$ :

$$
H_{K, V}(\lambda):=A \otimes I+I \otimes H_{\mathrm{b}, V}+\lambda \sum_{j=1}^{J} B_{j} \otimes \phi\left(g_{j, K, V}\right) .
$$

As in the case of $T_{j}$, one can show that $\left\{B_{j} \otimes \phi\left(i g_{j, K, V} / \omega_{V}\right)\right\}_{j=1}^{J}$ is a family of strongly commuting self-adjoint operators. Hence

$$
T_{K, V}:=\overline{\sum_{j=1}^{J} B_{j} \otimes \phi\left(i \frac{g_{j, K, V}}{\omega_{V}}\right)}
$$

is self-adjoint. We set

$$
U_{K, V}(\lambda):=e^{-i \lambda T_{K, V}}
$$

Let

$$
\begin{aligned}
& A_{K, V}(\lambda):=A-\frac{\lambda^{2}}{2} \sum_{j, l=1}^{J}\left\langle\frac{g_{j, K, V}}{\sqrt{\omega_{V}}}, \frac{g_{l, K, V}}{\sqrt{\omega_{V}}}\right\rangle B_{j} B_{l}, \\
& L_{K, V}(\lambda):=A_{K, V}(\lambda) \otimes I+I \otimes H_{\mathrm{b}, V}, \\
& \delta A_{1, K, V}(\lambda):=U_{K, V}(\lambda) A_{1} \otimes I U_{K, V}(\lambda)^{-1}-A_{1} \otimes I .
\end{aligned}
$$

and

$$
\widetilde{H}_{K, V}(\lambda):=L_{K, V}(\lambda)+\delta A_{1, K, V}(\lambda)
$$


Lemma 4.1 For all $\lambda \in \mathbb{R}$,

$$
U_{K, V}(\lambda) D(L)=D(L)
$$

and, for all $\Psi \in D(L)$,

$$
U_{K, V}(\lambda) H_{K, V}(\lambda) U_{K, V}(\lambda)^{-1}=\widetilde{H}_{K, V}(\lambda) \Psi
$$

Proof. By [4, Lemma 3.1], we have

$$
D\left(H_{\mathrm{b}, V}\right)=D\left(H_{\mathrm{b}}\right)
$$

which implies that

$$
D\left(A \otimes I+I \otimes H_{\mathrm{b}, V}\right)=D(L) .
$$

Then, in a way similar to the proof of Lemma 3.7, one can prove (4.13) and (4.14).

Lemma 4.2 For all sufficiently large $V, D(L) \subset D\left(\delta A_{1, K, V}(\lambda)\right)$ and, for all $\Psi \in D(L)$,

$$
\left\|\delta A_{1, K, V}(\lambda) \Psi\right\| \leq|\lambda|\left(c_{3 / 2, K, V}(g)\left\|I \otimes H_{\mathrm{b}, V}^{1 / 2} \Psi\right\|+\frac{1}{2} c_{1, K, V}(g)\|\Psi\|\right),
$$

where $c_{s, K, V}(g)$ is the constant $c_{s}(g)$ with $g_{j}$ (resp. $\left.\omega\right)$ replaced by $g_{j, K, V}$ (res. $\left.\omega_{V}\right)$.

Proof. Similar to the proof of Lemma 3.10.

Let

$$
g_{j, K}(k):=\chi_{[-K, K]}\left(k_{1}\right) \cdots \chi_{[-K, K]}\left(k_{d}\right) g_{j}(k), \quad k \in \mathbb{R}^{d}, j=1, \cdots, J .
$$

Then the following hold [4, Lemma 3.2]:

$$
\begin{aligned}
& \lim _{V \rightarrow \infty}\left\|g_{j, K, V}-g_{j, K}\right\|=0, \\
& \lim _{K \rightarrow \infty}\left\|g_{j, K}-g_{j}\right\|=0, \\
& \lim _{V \rightarrow \infty}\left\|\frac{g_{j, K, V}}{\sqrt{\omega_{V}}}-\frac{g_{j, K}}{\sqrt{\omega}}\right\|=0, \\
& \lim _{K \rightarrow \infty}\left\|\frac{g_{j, K}}{\sqrt{\omega}}-\frac{g_{j}}{\sqrt{\omega}}\right\|=0 .
\end{aligned}
$$

Let

$$
A_{K}(\lambda):=A-\frac{\lambda^{2}}{2} \sum_{j, l=1}^{J}\left\langle\frac{g_{j, K}}{\sqrt{\omega}}, \frac{g_{l, K}}{\sqrt{\omega}}\right\rangle B_{j} B_{l} .
$$

Lemma 4.3 Let $\lambda \in \Lambda$. Then, there exists a constant $K_{0}(\lambda)>0$ such that, for all $K>K_{0}(\lambda), A_{K}(\lambda)$ is self-adjoint with $D\left(A_{K}(\lambda)\right)=D\left(A_{0}\right)$ and bounded from below. 
Proof. We write

$$
A_{K}(\lambda)=A(\lambda)+\lambda^{2} D_{K}
$$

where

$$
D_{K}:=\frac{1}{2} \sum_{j, l=1}^{J} c_{j, l}(K, V) B_{j} B_{l}
$$

with

$$
c_{j, l}(K):=\left\langle\frac{g_{j}}{\sqrt{\omega}}, \frac{g_{l}}{\sqrt{\omega}}\right\rangle-\left\langle\frac{g_{j, K}}{\sqrt{\omega}}, \frac{g_{l, K}}{\sqrt{\omega}}\right\rangle .
$$

By $(2.21)$,

$$
\left\|B_{j} B_{l} u\right\| \leq\left(c_{j} c_{l}+\varepsilon\right)\left\|A_{0} u\right\|+\left(\frac{\left(c_{j} d_{l}+c_{l} d_{j}\right)^{2}}{4 \varepsilon}+d_{j} d_{l}\right)\|u\|, \quad u \in D\left(A_{0}\right),
$$

where $\varepsilon>0$ is arbitrary. Since $A(\lambda)$ is self-adjoint with $D(A(\lambda))=D\left(A_{0}\right)$, it follows from the closed graph theorem that there exists constant $\nu(\lambda)>0$ and $\mu(\lambda)>0$ such that, for all $u \in D\left(A_{0}\right)$,

$$
\left\|A_{0} u\right\| \leq \nu(\lambda)\|A(\lambda) u\|+\mu(\lambda)\|u\|
$$

Hence, for all $u \in D\left(A_{0}\right)$,

$$
\left\|D_{K} u\right\| \leq \alpha_{K}(\lambda)\|A(\lambda) u\|+\beta_{K}(\lambda)\|u\|,
$$

where

$$
\begin{aligned}
\alpha_{K}(\lambda) & :=\frac{\nu(\lambda)}{2} \sum_{j, l=1}^{J}\left|c_{j, l}(K)\right|\left(c_{j} c_{l}+\varepsilon\right), \\
\beta_{K}(\lambda) & :=\frac{\mu(\lambda)}{2} \sum_{j, l=1}^{J}\left|c_{j, l}(K)\right|\left(\frac{\left(c_{j} d_{l}+c_{l} d_{j}\right)^{2}}{4 \varepsilon}+d_{j} d_{l}\right) .
\end{aligned}
$$

By (4.22), $\lim _{K \rightarrow \infty} \alpha_{K}(\lambda)=0$. Hence there exists a constant $K_{0}(\lambda)>0$ such that, for all $K>K_{0}(\lambda), \lambda^{2} \alpha_{K}(\lambda)<1$. Then, by the Kato-Rellich theorem, $A_{K}(\lambda)$ is self-adjoint with $D\left(A_{K}(\lambda)\right)=D(A(\lambda))=D\left(A_{0}\right)$ and bounded from below.

In what follows we assume that $K>K_{0}(\lambda)(\lambda \in \Lambda)$.

Lemma 4.4 Let $\lambda \in \Lambda$. Then, for all sufficiently large $V, A_{K, V}(\lambda)$ is self-adjoint with $D\left(A_{K, V}(\lambda)\right)=D\left(A_{0}\right)$ and bounded from below.

Proof. We write

$$
A_{K, V}(\lambda)=A_{K}(\lambda)+\lambda^{2} D_{K, V}
$$

where

$$
D_{K, V}:=\frac{1}{2} \sum_{j, l=1}^{J} c_{j, l}(K, V) B_{j} B_{l}
$$

with

$$
c_{j, l}(K, V):=\left\langle\frac{g_{j, K}}{\sqrt{\omega}}, \frac{g_{l, K}}{\sqrt{\omega}}\right\rangle-\left\langle\frac{g_{j, K, V}}{\sqrt{\omega_{V}}}, \frac{g_{l, K, V}}{\sqrt{\omega_{V}}}\right\rangle .
$$


In the same way as in the proof of Lemma 4.3 [use (4.21)], we can show that $\lambda^{2} D_{K, V}$ is relatively bounded w.r.t. $A_{K}(\lambda)$ with relative bound small than 1 for all sufficiently large $V$. Thus, by the Kato-Rellich theorem, the assertion holds.

Lemma 4.5 Let $\lambda \in \Lambda$. Then, for all sufficiently large $V>0, \widetilde{H}_{K, V}(\lambda)$ and $H_{K, V}(\lambda)$ are self-adjoint with $D\left(\widetilde{H}_{K, V}(\lambda)\right)=D\left(H_{K, V}(\lambda)\right)=D\left(A_{0} \otimes I\right) \cap D\left(I \otimes H_{\mathrm{b}}\right)$ and bounded from below.

Proof. Similar to the proof of Theorem 2.2.

The following fact is well-known:

Lemma 4.6 The operator $H_{\mathrm{b}, V}$ is reduced by $\mathcal{F}_{\mathrm{b}}\left(\mathcal{W}_{V}\right)$ and its reduced part is equal to the second quantization of $\omega \mid \mathcal{W}_{V}$ in $\mathcal{F}_{\mathrm{b}}\left(\mathcal{W}_{V}\right)$.

Let

$$
\mathcal{F}_{V}:=\mathcal{H} \otimes \mathcal{F}_{\mathrm{b}}\left(\mathcal{W}_{V}\right)
$$

Then we have the orthogonal decomposition

$$
\mathcal{F}=\mathcal{F}_{V} \oplus \mathcal{F}_{V}^{\perp}
$$

where

$$
\left.\mathcal{F}_{V}^{\perp}=\oplus_{n=1}^{\infty} \mathcal{F}_{V} \otimes\left[\otimes_{\mathrm{s}}^{n} \mathcal{W}_{V}^{\perp}\right)\right] .
$$

Lemma 4.7 The operator $\widetilde{H}_{K, V}(\lambda)$ is reduced by $\mathcal{F}_{V}$ and

$$
\widetilde{H}_{K, V}(\lambda) \mid \mathcal{F}_{V}^{\perp} \geq E_{0}\left(H_{K, V}(\lambda)\right)+m
$$

Proof. It is easy to see that $g_{j, K, V} / \omega_{V} \in \mathcal{W}_{V}$. Hence, under the identifications (4.29) and (4.30), we have $B_{j} \otimes \phi\left(i g_{j, K, V} / \omega_{V}\right)=\left[B \otimes \phi\left(i g_{j, K, V} / \omega_{V}\right)\right] \oplus 0$. It follows that $T_{K, V}$ is reduced by $\mathcal{F}_{V}$ and so is $U_{K, V}(\lambda)$, which implies that $\delta A_{1, K, V}(\lambda)$ is reduced by $\mathcal{F}_{V}$. By this fact and Lemma $4.6, \widetilde{H}_{K, V}(\lambda)$ is reduced by $\mathcal{F}_{V}$. Then a method similar to the proof of $[4$, Lemma 3.10] yields (4.32).

Let

$$
\Sigma_{\lambda, K}:=\inf \sigma_{\text {ess }}\left(A_{K}(\lambda)\right)
$$

Lemma 4.8 There exists a constant $\varepsilon_{K}>0$ such that $\lim _{K \rightarrow \infty} \varepsilon_{K}=0$ and

$$
\Sigma_{\lambda} \leq \Sigma_{\lambda, K}+\varepsilon_{K}
$$

Proof. By (4.27) and a general theorem [16, p.168, Theorem X.18], one can show that

$$
\left(1-a_{K}\right) A(\lambda)-\left[\mu-E_{0}(A(\lambda))\right] a_{K} \leq A_{K}(\lambda) \leq\left(1+a_{K}\right) A(\lambda)+\left[\mu-E_{0}(A(\lambda))\right] a_{K}
$$

with

$$
a_{K}:=\lambda^{2}\left\{\alpha_{K}(\lambda)\left(1+\frac{\left|E_{0}(A(\lambda))\right|}{\mu}\right)+\frac{\beta_{K}(\lambda)}{\mu}\right\}
$$


where $\mu>0$ is arbitrary. For all sufficiently large $K$, we have $1-a_{K}>0$. We fix such a $K$. Then it follows from the first inequality in (4.34) and the min-max principle [17, p.76, Theorem XIII.1] that

$$
\left(1-a_{K}\right) \Sigma_{\lambda} \leq \Sigma_{\lambda, K}+\left[\mu-E_{0}(A(\lambda))\right] a_{K},
$$

which implies that

$$
\Sigma_{\lambda} \leq \Sigma_{\lambda, K}+\varepsilon_{K}
$$

with $\varepsilon_{K}:=a_{K}\left[\Sigma_{\lambda}+\mu-E_{0}(A(\lambda))\right]$. We have $\lim _{K \rightarrow \infty} \varepsilon_{K}=0$.

Let

$$
\Sigma_{\lambda, K, V}:=\inf \sigma_{\text {ess }}\left(A_{K, V}(\lambda)\right)
$$

Lemma 4.9 There exists a constant $\eta_{K, V}>0$ such that $\lim _{V \rightarrow \infty} \eta_{K, V}=0$ and

$$
\Sigma_{\lambda, K} \leq \Sigma_{\lambda, K, V}+\eta_{K, V}
$$

Proof. Similar to the proof of Lemma 4.8.

Lemma 4.10

$$
\begin{aligned}
& \lim _{K \rightarrow \infty} E_{0}\left(A_{K}(\lambda)\right)=E_{0}(A(\lambda)), \\
& \lim _{V \rightarrow \infty} E_{0}\left(A_{K, V}(\lambda)\right)=E_{0}\left(A_{K}(\lambda)\right) .
\end{aligned}
$$

Proof. By (4.34) and the variational principle,

$$
\left(1-a_{K}\right) E_{0}(A(\lambda))-\mu a_{K} \leq E_{0}\left(A_{K}(\lambda)\right) \leq\left(1+a_{K}\right) E_{0}(A(\lambda))+\mu a_{K},
$$

which implies (4.36). Similarly one can prove (4.37).

Lemma 4.11 Suppose that the same hypothesis as in Theorem 2.3 and Hypothesis VIII hold. Then, for all sufficiently large $K$ and $V, H_{K, V}(\lambda)$ has purely discrete spectrum in $\left[E_{0}\left(H_{K, V}(\lambda)\right), E_{0}\left(H_{K, V}(\lambda)\right)+m\right)$.

Proof. By Lemma 4.1, we need only to show that $\widetilde{H}_{K, V}(\lambda)$ has purely discrete spectrum in $\left[E_{0}\left(H_{K, V}(\lambda)\right), E_{0}\left(H_{K, V}(\lambda)\right)+m\right)$ [note that $\left.E_{0}\left(h_{K, V}\right)=E_{0}\left(\widetilde{H}_{K, V}(\lambda)\right)=E_{0}\left(H_{K, V}(\lambda)\right)\right]$. By Lemma 4.7 , it is sufficient to show that the reduced part $h_{K, V}:=\widetilde{H}_{K, V}(\lambda) \mid \mathcal{F}_{V}$ has such a property. By Lemma 4.2, we have

$$
\left|\left\langle\Psi, \delta A_{1, K, V}(\lambda) \Psi\right\rangle\right| \leq \varepsilon\left\langle\Psi, I \otimes H_{\mathrm{b}, V} \Psi\right\rangle+b_{\varepsilon}\|\Psi\|^{2}, \Psi \in D\left(I \otimes H_{\mathrm{b}, V}\right),
$$

where $\varepsilon>0$ is arbitrary and

$$
b_{\varepsilon}:=\left(\frac{\lambda^{2} c_{3 / 2, K, V}(g)^{2}}{4 \varepsilon}+\frac{1}{2}|\lambda| c_{1, K, V}(g)\right) .
$$


By condition (2.25) and Lemmas 4.8-4.10, we have

$$
\Sigma_{\lambda, K, V}-E_{0}\left(A_{K, V}(\lambda)\right)>m+2 b_{\varepsilon}
$$

if $\varepsilon<1$ is sufficiently close to 1 and $K$ and $V$ are sufficiently large. Note that the spectrum of $h_{0}:=H_{\mathrm{b}, V} \mid \mathcal{F}_{\mathrm{b}}\left(\mathcal{W}_{V}\right)$ is purely discrete with $\operatorname{Ran}\left(E_{h_{0}}([0, s])\right.$ being finite dimensional for all $s>0$. Hence we can apply Theorem B.3 with Remark B.1 in Appendix B to conclude that $h_{K, V}$ has purely discrete spectrum in $\left[E_{0}\left(h_{K, V}\right), E_{0}\left(h_{K, V}\right)+m\right)$.

Proof of Theorem 2.3

Let

$$
H_{K}(\lambda):=A \otimes I+I \otimes H_{\mathrm{b}}+\lambda \sum_{j=1}^{J} B_{j} \otimes \phi\left(g_{j, K}\right) .
$$

Then, in the same way as in [4, Lemma 3.5], one can show that $H_{K, V}(\lambda)$ converges to $H_{K}(\lambda)$ in the norm resolovent sense as $V \rightarrow \infty$. Hence, by Lemma 4.11 and an application of [4, Lemma 3.12], we conclude that $H_{K}(\lambda)$ has purely discrete spectrum in $\left[E_{0}\left(H_{K}(\lambda)\right), E_{0}\left(H_{K}(\lambda)\right)+m\right)$.

In the same way as in [4, Lemma 3.11], one can show that $H_{K}(\lambda)$ converges to $H(\lambda)$ in the norm resolovent sense as $K \rightarrow \infty$. Hence, by the preceding result and [4, Lemma 3.12] again, we see that $H(\lambda)$ has purely discrete spectrum in $\left[E_{0}(H(\lambda)), E_{0}(H(\lambda))+m\right)$.

Finally we consider the case where each $g_{j}$ is not necessarily continuous. In this case we can take a sequence of continuous functions $\left\{g_{j}^{(n)}\right\}_{n=1}^{\infty}$ such that $g_{j}^{(n)} \in \mathcal{W}$ such that $\left\|g_{j}^{(n)}-g_{j}\right\| \rightarrow 0(n \rightarrow \infty)$. Let $H_{n}$ be the operator $H(\lambda)$ with $g_{j}$ replaced $g_{j}^{(n)}$ $(j=1, \cdots, J)$. Then one can show that $H_{n}$ converges to $H(\lambda)$ in the norm resolovent sense as $n \rightarrow \infty$. By the result of the last paragraph, $H_{n}$ has purely discrete spectrum in $\left[E_{0}\left(H_{n}\right), E_{0}\left(H_{n}\right)+m\right)$. Hence, by [4, Lemma 3.12] once again, we see that $H(\lambda)$ has purely discrete spectrum in $\left[E_{0}(H(\lambda)), E_{0}(H(\lambda))+m\right)$.

\section{Existence of a Ground State in the Massless Case}

This section is devoted to proof of Theorem 2.4. Throughout the section, all the hypotheses of Theorem 2.4 are assumed. For each constant $M>0$, we define $\omega_{M}: \mathbb{R}^{d} \rightarrow[M, \infty)$ by

$$
\omega_{M}(k):=\omega(k)+M, \quad k \in \mathbb{R}^{d},
$$

so that $\inf _{k \in \mathbb{R}^{d}} \omega_{M}(k)=M>0$. We set

$$
g_{j}^{(M)}:=\frac{\omega_{M}}{\omega} g_{j},
$$

which is in $\mathcal{W}$. We introduce a "regularized" version of the Hamiltonian $H(\lambda)$ :

$$
H_{M}:=A \otimes I+I \otimes H_{\mathrm{b}, M}+\lambda \sum_{j=1}^{J} B_{j} \otimes \phi\left(g_{j}^{(M)}\right),
$$

where

$$
H_{\mathrm{b}, M}:=d \Gamma\left(\omega_{M}\right)
$$


Let

$$
A_{M}:=A-\lambda^{2} R_{B}^{(M)}
$$

with

$$
R_{B}^{(M)}:=\frac{1}{2} \sum_{j, l=1}^{J}\left\langle\frac{g_{j}^{(M)}}{\sqrt{\omega_{M}}}, \frac{g_{l}^{(M)}}{\sqrt{\omega_{M}}}\right\rangle B_{j} B_{l}
$$

and

$$
\widetilde{H}_{M}:=A_{M} \otimes I+I \otimes H_{\mathrm{b}, M}+\delta A_{1}(\lambda) .
$$

Then, by Lemma 3.7,

$$
U(\lambda) H_{M} U(\lambda)^{-1}=\widetilde{H}_{M} .
$$

By applying the Lebesgue dominated convergence theorem, one can show that

$$
\lim _{M \rightarrow 0}\left\|\frac{g_{j}^{(M)}}{\omega_{M}^{s}}-\frac{g_{j}}{\omega^{s}}\right\|=0
$$

for all $s \geq 0$ such that $g_{j} / \omega^{s+1} \in \mathcal{W}$. We write

$$
A_{M}=A(\lambda)+W_{M}
$$

with

$$
W_{M}:=\lambda^{2}\left(R_{B}-R_{B}^{(M)}\right) .
$$

We put

$$
c_{M}:=\lambda^{2} \sum_{j, l=1}^{J}\left|\left\langle\frac{g_{j}}{\sqrt{\omega}}, \frac{g_{l}}{\sqrt{\omega}}\right\rangle-\left\langle\frac{g_{j}^{(M)}}{\sqrt{\omega_{M}}}, \frac{g_{l}^{(M)}}{\sqrt{\omega_{M}}}\right\rangle\right| .
$$

Then we can show that

$$
\left\|W_{M} u\right\| \leq c_{M}(a\|A(\lambda) u\|+b\|u\|), \quad u \in D\left(A_{0}\right),
$$

where $a$ and $b$ are constants independent of $M$ (cf. the proof of Lemma 4.3). In the same way as in Lemma 4.10, one can show that

$$
\lim _{M \rightarrow 0} E_{0}\left(A_{M}\right)=E_{0}(A(\lambda)) .
$$

By this fact, (5.1) and (2.26), we can take $M>0$ (sufficiently small) satisfying

$$
\Sigma_{\lambda}-E_{0}\left(A_{M}\right)>M+\frac{1}{2} \lambda^{2} c_{3 / 2}^{(M)}(g)^{2}+|\lambda| c_{1}^{(M)}(g),
$$

where $c_{s}^{(M)}(g)$ is the $c_{s}(g)$ with $\omega$ and $g_{j}$ replaced by $\omega_{M}$ and $g_{j}^{(M)}$ respectively. It follows from Theorem 2.3 that $H_{M}$ has a ground state and so does $\widetilde{H}_{M}$. We denote a normalized ground state of $\widetilde{H}_{M}$ by $\Psi_{M}$.

Lemma 5.1 For all $f \in \mathcal{W}$ with $\omega f \in \mathcal{W}, I \otimes a(f) \Psi_{M} \in D\left(\widetilde{H}_{M}\right)$ and

$$
\begin{aligned}
& \left(\widetilde{H}_{M}-E_{0}\left(\widetilde{H}_{M}\right)\right) I \otimes a(f) \Psi_{M} \\
& =\left\{-a\left(\omega_{M} f\right)-\sum_{j=1}^{J} \frac{\lambda}{\sqrt{2}}\left\langle f, \frac{g_{j}}{\omega}\right\rangle U(\lambda)\left[B_{j}, A_{1}\right] U(\lambda)^{-1}\right\} \Psi_{M} .
\end{aligned}
$$


Proof. Similar to the proof of [4, Lemma 4.1] except that, in the present case, one uses an easily proven formula

$$
U(\lambda) I \otimes a(f) U(\lambda)^{-1}=I \otimes a(f)-\frac{\lambda}{\sqrt{2}}\left\langle f, \frac{g_{j}}{\omega}\right\rangle B_{j} \otimes I
$$

on $D\left(A_{0} \otimes I\right) \cap D\left(I \otimes H_{\mathrm{b}, M}\right)$.

We set

$$
N_{\mathrm{b}}:=d \Gamma(I)
$$

the number operator on $\mathcal{F}_{\mathrm{b}}(\mathcal{W})$. Then we have for all $f \in \mathcal{W}$ and $\psi \in D\left(N_{\mathrm{b}}^{1 / 2}\right)$

$$
\|a(f) \psi\| \leq\|f\|\left\|N_{\mathrm{b}}^{1 / 2} \psi\right\|, \quad\left\|a(f)^{*} \psi\right\| \leq\|f\|\left\|N_{\mathrm{b}}^{1 / 2} \psi\right\|+\|f\|,
$$

which implies that

$$
\|\phi(f) \psi\| \leq \sqrt{2}\|f\|\left\|N_{\mathrm{b}}^{1 / 2} \psi\right\|+\frac{1}{\sqrt{2}}\|f\|\|\psi\| .
$$

Now we can apply Theorem B.4 in Appendix B with $(K, B, C)=\left(A(\lambda), H_{\mathrm{b}}, \delta A_{1}(\lambda)\right)$ so that $H=\widetilde{H}(\lambda)$. By (2.25), Hypothesis (B.1) holds. Hypothesis (B.2) is satisfied with $m=M, K_{m}=A_{M}, B_{m}=H_{\mathrm{b}, M}, \psi_{0}=\Omega$ (the Fock vacuum) and $D=\mathcal{F}_{0}(\mathcal{W}) \cap D\left(H_{\mathrm{b}}\right)$. We denote by $P_{\Omega}$ the orthogonal projection onto the one-dimensional subspace $\{\alpha \Omega \mid \alpha \in \mathbb{C}\}$ and set $P_{\Omega}^{\perp}$.: $=I-P_{\Omega}$. By (3.23) and the fact that $H_{\mathrm{b}, M} \Omega=0$ and $H_{\mathrm{b}} \Omega=0$, we have for all $u \in D\left(A_{0}\right)$ with $\|u\|=1$

$$
\left\langle u \otimes \Omega, \widetilde{H}_{M} u \otimes \Omega\right\rangle \leq\left\langle u, A_{M} u\right\rangle+\frac{|\lambda|}{2} c_{1}(g),
$$

which implies that $E_{0}\left(\widetilde{H}_{M}\right) \leq E_{0}\left(A_{M}\right)+|\lambda| c_{1}(g) / 2$. By $(5.2)$, for every $\eta>0$, there exists a constant $M_{0}>0$ such that $\left|E_{0}\left(A_{M}\right)-E_{0}(A(\lambda))\right|<\eta$ for all $M<M_{0}$. By this fact and (2.26), we have

$$
E_{0}\left(\widetilde{H}_{M}\right) \leq \eta+E_{0}(A(\lambda))+\frac{|\lambda|}{2} c_{1}(g)<\Sigma_{\lambda}
$$

for all $M<M_{0}$, where we take $\eta$ sufficiently small. Thus, if we show that

$$
\frac{1}{\left(\Sigma_{\lambda}-E_{0}\left(\widetilde{H}_{M}\right)\right)^{2}}\left\|\delta A_{1}(\lambda) \Psi_{M}\right\|^{2}+\left\|I \otimes P_{\Omega}^{\perp} \Psi_{M}\right\|^{2}<\delta
$$

for some $\delta<1$, then $\widetilde{H}(\lambda)$ has a ground state and so does $H(\lambda)$.

Let us prove (5.5). Using estimate (5.4) and (3.24), we have

$$
\left\|\delta A_{1}(\lambda) \Psi_{M}\right\| \leq|\lambda| c_{1}(g)\left\|N_{\mathrm{b}}^{1 / 2} \Psi_{M}\right\|+\frac{|\lambda| c_{1}(g)}{2} .
$$

It is well known or easy to see that $N_{\mathrm{b}} \geq P_{\Omega}^{\perp}$. Hence

$$
\left\|I \otimes P_{\Omega}^{\perp} \Psi_{M}\right\|^{2} \leq\left\|N_{\mathrm{b}}^{1 / 2} \Psi_{M}\right\|^{2} .
$$


Therefore, if

$$
\left(\frac{2|\lambda|^{2} c_{1}(g)^{2}}{\left[\Sigma_{\lambda}-E_{0}\left(\widetilde{H}_{M}\right)\right]^{2}}+1\right)\left\|N_{\mathrm{b}}^{1 / 2} \Psi_{M}\right\|^{2}+\frac{|\lambda|^{2} c_{1}(g)^{2}}{\left[\Sigma_{\lambda}-E_{0}\left(\widetilde{H}_{M}\right)\right]^{2}}<\delta
$$

then (5.5) follows.

To estimate $\left\|N_{\mathrm{b}}^{1 / 2} \Psi_{M}\right\|$,we follow the method given in the proof of [4, Lemma 4.3]. Indeed, by Lemma 5.1, we can show that

$$
\left\|N_{\mathrm{b}}^{1 / 2} \Psi_{M}\right\| \leq \frac{|\lambda|}{\sqrt{2}}\left(\sum_{j=1}^{J}\left\|\frac{g_{j}}{\omega \omega_{M}}\right\|\left\|\left[B_{j}, A_{1}\right]\right\|\right) .
$$

Hence, if

$$
\left(\frac{2|\lambda|^{2} c_{1}(g)^{2}}{\left[\Sigma_{\lambda}-E_{0}\left(\widetilde{H}_{M}\right)\right]^{2}}+1\right) \frac{\lambda^{2}}{2}\left(\sum_{j=1}^{J}\left\|\frac{g_{j}}{\omega \omega_{M}}\right\|\left\|\left[B_{j}, A_{1}\right]\right\|\right)^{2}+\frac{|\lambda|^{2} c_{1}(g)^{2}}{\left[\Sigma_{\lambda}-E_{0}\left(\widetilde{H}_{M}\right)\right]^{2}}<\delta,
$$

then (5.6) follows. In the same way as in [4, Lemma 4.11], we can show that

$$
\lim _{M \rightarrow 0} E_{0}\left(H_{M}\right)=E_{0}(H(\lambda))
$$

We have $E_{0}\left(H_{M}\right)=E_{0}\left(\widetilde{H}_{M}\right)$. Hence condition (2.27) implies (5.7) with $M>0$ sufficiently small. This completes the proof of Theorem 2.4 .

\section{The Pauli-Fierz Type Model}

In this section, we apply Theorem 2.4 to a model of the Pauli-Fierz type in non-relativistic QED . Namely we consider the case where the system $\mathrm{S}$ is a system of $n$ non-relativistic quantum particles moving in $\mathbb{R}^{d}$ under the influence of a potential $V: \mathbb{R}^{d n} \rightarrow \mathbb{R}(d, n \in$ $\mathbb{N})$. We set $\nu:=n d$. We assume for simplicity that

$$
\nu \geq 3, \quad V \in C_{0}^{\infty}\left(\mathbb{R}^{\nu}\right), \quad V_{-}:=\min \{V, 0\} \neq 0
$$

The Hilbert space of the particle system is taken to be

$$
\mathcal{H}=L^{2}\left(\mathbb{R}^{\nu}\right)
$$

Hence the Hamiltonian of the particle system is

$$
H_{\mathrm{p}}:=-\Delta+\alpha V
$$

acting in $L^{2}\left(\mathbb{R}^{\nu}\right)$, where $\Delta$ is the generalized Laplacian on $L^{2}\left(\mathbb{R}^{\nu}\right)$ and $\alpha>0$ is a parameter. We write $x=\left(x_{1}, \cdots, x_{\nu}\right) \in \mathbb{R}^{\nu}$ and define

$$
p_{j}:=-i D_{j}
$$


with $D_{j}$ being the generalized partial differential operator in the variable $x_{j}$. By the Cwikel-Lieb-Rosenbljum bound [17, Theorem XIII.12], $H_{\mathrm{p}}$ has no ground state for all sufficiently small $\alpha$.

Let $g_{j}: \mathbb{R}^{d} \rightarrow \mathbb{R}^{N}(j=1, \cdots, \nu)$ be such that

$$
g_{j}, \quad \frac{g_{j}}{\omega^{2}} \in \mathcal{W} .
$$

We take as the total Hamiltonian of the composed system

$$
H_{\mathrm{PF}}(\lambda):=H_{\mathrm{p}} \otimes I+I \otimes H_{\mathrm{b}}+\lambda \sum_{j=1}^{\nu} p_{j} \otimes \phi\left(g_{j}\right) .
$$

This model is a concrete realization of the abstract model $H(\lambda)$ with the following choice:

$$
A_{0}=-\Delta, \quad A_{1}=\alpha V, \quad J=\nu, \quad B_{j}=p_{j} .
$$

It is straightforward to see that Hypotheses I-V hold with

$$
\left[B_{j}, A_{1}\right] \mid D\left(A_{0}\right)=-i \alpha D_{j} V .
$$

Suppose that, for all $\xi=\left(\xi_{1}, \cdots, \xi_{\nu}\right) \in \mathbb{R}^{\nu}$,

$$
\frac{1}{2} \sum_{j, l=1}^{\nu}\left\langle\frac{g_{j}}{\sqrt{\omega}}, \frac{g_{l}}{\sqrt{\omega}}\right\rangle \xi_{j} \xi_{l}=G(g) \xi^{2}
$$

with $G(g)>0$ a constant independent of $\xi$. This condition is satisfied in the original Pauli-Fierz model without $A^{2}$-term in the dipole approximation [2] (see Example 6.2 below). Condition (6.2) implies that

$$
\frac{1}{2} \sum_{j, l=1}^{\nu}\left\langle\frac{g_{j}}{\sqrt{\omega}}, \frac{g_{l}}{\sqrt{\omega}}\right\rangle p_{j} p_{l}=-G(g) \Delta .
$$

Hence, in the present case, $A(\lambda)$ takes the following form:

$$
A(\lambda)=-\left(1-\lambda^{2} G(g)\right) \Delta+\alpha V
$$

Therefore, in the present case,

$$
\Lambda=(-\lambda(g), 0) \cup(0, \lambda(g)) \neq \emptyset,
$$

where

$$
\lambda(g):=\frac{1}{\sqrt{G(g)}} .
$$

Thus Hypothesis VI holds.

Also we have

$$
c_{s}(g)=\sqrt{2}|\alpha| \sum_{j=1}^{\nu}\left\|D_{j} V\right\|_{\infty}\left\|\frac{g_{j}}{\omega^{s}}\right\|,
$$

where $\|F\|_{\infty}:=\sup _{x \in \mathbb{R}^{\nu}}|F(x)|\left(F: \mathbb{R}^{\nu} \rightarrow \mathbb{C}\right)$.

We set

$$
V_{0}:=\inf _{x \in \mathbb{R}^{\nu}} V(x)<0 .
$$

We first consider the massive case. 
Theorem 6.1 Consider the case $m>0$. Let $\nu \geq 3$ and Hypothesis VII be satified. Suppose that

$$
\alpha\left|V_{0}\right|>\frac{1}{2} \lambda(g)^{2} c_{3 / 2}(g)^{2}+\lambda(g) c_{1}(g)
$$

and the constant $m$ satisfies

$$
\alpha\left|V_{0}\right|>m+\frac{1}{2} \lambda(g)^{2} c_{3 / 2}(g)^{2}+\lambda(g) c_{1}(g) .
$$

Then there exists a constant $\delta$ such that, for all $|\lambda| \in(\lambda(g)-\delta, \lambda(g)), H_{\mathrm{PF}}(\lambda)$ has purely discrete spectrum in the interval $\left[E_{0}\left(H_{\mathrm{PF}}(\lambda)\right), E_{0}\left(H_{\mathrm{PF}}(\lambda)\right)+m\right)$. In particular, $H_{\mathrm{PF}}(\lambda)$ has a ground state.

Proof. Let $0<|\lambda|<\lambda(g)$. By [17, Theorem XIII.15],

$$
\Sigma_{\lambda}=\inf \sigma_{\text {ess }}(A(\lambda))=0 .
$$

Therefore, by Theorem 2.3, we need only to show

$$
-E_{0}(A(\lambda))>m+\frac{1}{2} \lambda^{2} c_{3 / 2}(g)^{2}+|\lambda| c_{1}(g)
$$

for all $|\lambda|$ sufficiently close to $\lambda(g)$. We can take a constant $\varepsilon>0$ such that $E:=$ $V_{0}+\varepsilon<0$. Then $D_{E}:=\left\{x \in \mathbb{R}^{\nu} \mid V(x)<E\right\}$ is a non-empty bounded open set. Let $d_{E}:=\inf _{u \in C_{0}^{\infty}\left(D_{E}\right),\|u\|=1}\langle u,-\Delta u\rangle$. Then, by the strict positivity of the Dirichlet Laplacian in a bounded open set, $d_{E}>0$. By the variational principle and the fact that $\langle u, V u\rangle \leq E\|u\|^{2}, u \in C_{0}^{\infty}\left(D_{E}\right)$,

$$
E_{0}(A(\lambda)) \leq\left(1-\lambda^{2} G(g)\right) d_{E}+\alpha E .
$$

Note that $-\alpha E=\alpha\left|V_{0}\right|-\alpha \varepsilon$. Hence, by (6.5), for all sufficiently small $\varepsilon>0$ and $|\lambda|$ sufficiently close to $\lambda(g)$,

$$
-\alpha E-\left(1-\lambda^{2} G(g)\right) d_{E}>m+\frac{1}{2} \lambda(g)^{2} c_{3 / 2}(g)^{2}+\lambda(g) c_{1}(g) .
$$

Hence

$$
\begin{aligned}
-E_{0}(A(\lambda)) & >m+\frac{1}{2} \lambda(g)^{2} c_{3 / 2}(g)^{2}+\lambda(g) c_{1}(g) \\
& >m+\frac{1}{2} \lambda^{2} c_{3 / 2}(g)^{2}+|\lambda| c_{1}(g) .
\end{aligned}
$$

Thus (6.6) holds.

We next consider the massless case.

Theorem 6.2 Consider the case $m=0$. Let $\nu \geq 3$. Assume Hypothesis VII and (6.4). Suppose in addition that

$$
\frac{4 \lambda(g)^{2} c_{1}(g)^{2}}{\alpha^{2} V_{0}^{2}}+\frac{1}{2}\left(\frac{8 \lambda(g)^{2} c_{1}(g)^{2}}{\alpha^{2} V_{0}^{2}}+1\right) \lambda(g)^{2} c_{2}(g)^{2}<1 .
$$

Then there exists a constant $\delta$ such that, for all $|\lambda| \in(\lambda(g)-\delta, \lambda(g)), H_{\mathrm{PF}}(\lambda)$ has a ground state. 
Proof. Let $0<|\lambda|<\lambda(g)$. By Theorem 2.4 and by the proof of Theorem 6.1, we need only to check that

$$
\frac{\lambda^{2} c_{1}(g)^{2}}{E_{0}\left(H_{\mathrm{PF}}(\lambda)\right)^{2}}+\frac{1}{2}\left(\frac{2 \lambda^{2} c_{1}(g)^{2}}{E_{0}\left(H_{\mathrm{PF}}(\lambda)\right)^{2}}+1\right) \lambda^{2} c_{2}(g)^{2}<1
$$

for all $|\lambda|$ sufficiently close to $\lambda(g)$. By (6.7) and Corollary 3.12, we have

$$
\begin{aligned}
E_{0}\left(H_{\mathrm{PF}}(\lambda)\right) & \leq\left(1-\lambda^{2} G(g)\right) d_{E}+\alpha E+\frac{\lambda(g)}{2} c_{1}(g) \\
& \leq\left(1-\lambda^{2} G(g)\right) d_{E}-\frac{\alpha}{2}\left|V_{0}\right|+\alpha \varepsilon
\end{aligned}
$$

where, in the last step, we have used (6.4). For all $|\lambda|$ sufficiently close to $\lambda(g)$ and sufficiently small $\varepsilon$, the right hand side of (6.11) is negative. Hence, if we show that

$$
\frac{\lambda^{2} c_{1}(g)^{2}}{\left[\frac{\alpha\left|V_{0}\right|}{2}-\alpha \varepsilon-\left(1-\lambda^{2} G(g)\right) d_{E}\right]^{2}}+\frac{1}{2}\left(\frac{2 \lambda^{2} c_{1}(g)^{2}}{\left[\frac{\alpha\left|V_{0}\right|}{2}-\alpha \varepsilon-\left(1-\lambda^{2} G(g)\right) d_{E}\right]^{2}}+1\right) \lambda^{2} c_{2}(g)^{2}<1
$$

for all $|\lambda|$ sufficiently close to $\lambda(g)$ and sufficiently small $\varepsilon$, then (6.10) follows. It is easy to see that (6.9) implies (6.12) for all $|\lambda|$ sufficiently close to $\lambda(g)$ and sufficiently small $\varepsilon>0$.

Remark 6.1 Theorems 6.1 and 6.2 give only sufficient conditions for $H_{\mathrm{PF}}(\lambda)$ to have a ground state with $|\lambda|$ in an "intermediate" region. Suppose that $H_{\mathrm{p}}$ has no ground state. Then it would be an interesting problem to investigate if there is a constant $\lambda_{0}>0$ such that, for all $|\lambda| \in\left(0, \lambda_{0}\right), H_{\mathrm{PF}}(\lambda)$ has no ground state. Unfortunately we have been unable to give an answer to this problem.

Example 6.1 Assume Hypothesis VII. Let $\kappa>0$ and $H_{\mathrm{PF}}^{\kappa}(\lambda)$ be the $H_{\mathrm{PF}}(\lambda)$ with $\omega$ replaced by $\kappa \omega$. Then conditions (6.4) and (6.9) take the following forms respectively:

$$
\begin{aligned}
& \alpha\left|V_{0}\right|>\frac{1}{2 \kappa^{2}} \lambda(g)^{2} c_{3 / 2}(g)^{2}+\frac{1}{\sqrt{\kappa}} \lambda(g) c_{1}(g), \\
& \frac{4 \lambda(g)^{2} c_{1}(g)^{2}}{\kappa \alpha^{2} V_{0}^{2}}+\frac{1}{2 \kappa^{3}}\left(\frac{8 \lambda(g)^{2} c_{1}(g)^{2}}{\kappa \alpha^{2} V_{0}^{2}}+1\right) \lambda(g)^{2} c_{2}(g)^{2}<1 .
\end{aligned}
$$

For a given $\alpha\left|V_{0}\right|$, these inequalities are satisfied if $\kappa$ is sufficiently large. Thus $H_{\mathrm{PF}}^{\kappa}(\lambda)$ has a ground state for all sufficiently large $\kappa$ and $|\lambda|<\sqrt{\kappa} \lambda(g)$ sufficiently close to $\sqrt{\kappa} \lambda(g)$. This result is somewhat analogous to the results by Hiroshima and Spohn [14, Lemma 3.3, Theorem 3.4], except that the regime of the coupling constant is different.

Example 6.2 Consider the original Pauli-Fierz model with one non-relativistic particle in $\mathbb{R}^{3}$ so that $n=1, d=3$ and $N=2$. We take $\omega(k)=|k|, k \in \mathbb{R}^{3}$, and the momentum cutoff function $g_{j}: \mathbb{R}^{3} \rightarrow \mathbb{R}^{2}(j=1,2,3)$ as

$$
g_{j}(k)=\left(\frac{\chi_{[\sigma, L]}(|k|)}{\sqrt{(2 \pi)^{3}|k|}} e_{j}^{(1)}(k), \frac{\chi_{[\sigma, L]}(|k|)}{\sqrt{(2 \pi)^{3}|k|}} e_{j}^{(2)}(k)\right),
$$


where $\chi_{[\sigma, L]}$ is the characteristic function of the interval $[\sigma, L](\sigma>0$ is an infrared cutoff and $L>\sigma$ is an ultraviolet cutoff) and $e^{(r)}=\left(e_{1}^{(r)}, e_{2}^{(r)}, e_{3}^{(r)}\right): \mathbb{R}^{3} \rightarrow \mathbb{R}^{3}(r=1,2)$ is Borel measurable such that

$$
\left\langle e^{(s)}(k), e^{(r)}(k)\right\rangle=\delta_{s r}, \quad\left\langle e^{(r)}(k), k\right\rangle=0, \quad r, s=1,2, \text { a.e. } k \in \mathbb{R}^{3} .
$$

By the identity

$$
\sum_{r=1}^{2} e_{j}^{(r)}(k) e_{l}^{(r)}(k)=\delta_{j l}-\frac{k_{j} k_{l}}{|k|^{2}}, \quad \text { a.e. } k
$$

and the easily proven fact that

$$
\int_{\mathbb{R}^{3}} f(|k|) k_{j} k_{l} d k=\frac{1}{3} \delta_{j l} \int_{\mathbb{R}^{3}} f(|k|) k^{2} d k
$$

for all $f:[0, \infty) \rightarrow \mathbb{C}$ such that $\int_{\mathbb{R}^{3}} f(|k|) k^{2} d k<\infty$, we can show that

$$
\begin{aligned}
\left\langle\frac{g_{j}}{\omega^{s}}, \frac{g_{l}}{\omega^{s}}\right\rangle & =\frac{2}{3(2 \pi)^{3}} \delta_{j l} \int_{\mathbb{R}^{3}} \frac{\chi_{[\sigma, L]}(|k|)}{|k|^{2 s+1}} d k \\
& =\left\{\begin{array}{ll}
\delta_{j l} \frac{8 \pi}{3(2 \pi)^{3}} \log \frac{L}{\sigma} \\
\delta_{j l} \frac{8 \pi}{3(2 \pi)^{3}} \frac{1}{2(1-s)}\left(L^{2(1-s)}-\sigma^{2(1-s)}\right) & ; s \neq 1
\end{array} .\right.
\end{aligned}
$$

Hence, in the present example, we have

$$
\begin{aligned}
& G(g)=\frac{4 \pi}{(2 \pi)^{3}}(L-\sigma), \quad \lambda(g)=\sqrt{\frac{(2 \pi)^{3}}{4 \pi}} \frac{1}{\sqrt{L-\sigma}}, \\
& c_{1}(g)=\sqrt{2}|\alpha|\left(\sum_{j=1}^{3}\left\|D_{j} V\right\|_{\infty}\right) \sqrt{\frac{8 \pi}{3(2 \pi)^{3}}} \sqrt{\log \frac{L}{\sigma}} \\
& c_{3 / 2}(g)=\sqrt{2}|\alpha|\left(\sum_{j=1}^{3}\left\|D_{j} V\right\|_{\infty}\right) \sqrt{\frac{8 \pi}{3(2 \pi)^{3}}} \sqrt{\frac{1}{\sigma}-\frac{1}{L}} \\
& c_{2}(g)=\sqrt{2}|\alpha|\left(\sum_{j=1}^{3}\left\|D_{j} V\right\|_{\infty}\right) \sqrt{\frac{8 \pi}{3(2 \pi)^{3}}} \frac{1}{\sqrt{2}} \sqrt{\frac{1}{\sigma^{2}}-\frac{1}{L^{2}}} .
\end{aligned}
$$

From these formulas, we see that

$$
\begin{aligned}
& \lambda(g) c_{1}(g) \sim \text { const. } \sqrt{\frac{\log L}{L}}, \\
& \lambda(g) c_{3 / 2}(g) \sim \text { const. } \frac{1}{\sqrt{L}}, \\
& \lambda(g) c_{2}(g) \sim \text { const. } \frac{1}{\sqrt{L}}
\end{aligned}
$$

as $L \rightarrow \infty$, where "const." denotes a constant independent of $L$ sufficiently large. Hence, for all sufficiently large $L$, all the assumptions of Theorem 6.2 are satisfied. Thus, in the present example, $H_{\mathrm{PF}}(\lambda)$ has a ground state for all sufficiently large $L$ and $|\lambda|<\lambda(g)$ sufficiently close to $\lambda(g)$. A possible physical picture of this result is that the coupling of non-relativistic quantum particles to photons with larger momenta makes higher the possibility for $H_{\mathrm{PF}}(\lambda)$ to have a ground state. 


\section{Appendix}

\section{A Weak Differentiability of a Heisenberg Operator}

Let $\mathcal{X}$ be a Hilbert space. Let $H$ be a self-adjoint operator and $S$ a symmetric operator on $\mathcal{X}$. Then the Heisenberg operator ("time evolution") of $S$ with respect to $H$ is defined by

$$
S(t):=e^{i t H} S e^{-i t H}, \quad t \in \mathbb{R} .
$$

Proposition A.1 Suppose that there exists a self-adjoint operator $K$ on $\mathcal{X}$ such that the following (K.1) and (K.2) hold:

(K.1) K strongly commutes with $H$.

(K.2) $D(K) \subset D(S)$ and there exist constants $a, b \geq 0$ such that

$$
\|S \psi\| \leq a\|K \psi\|+b\|\psi\|, \quad \psi \in D(K) .
$$

Then, for all $\psi, \phi \in D(K) \cap D(H)$, the function: $t \mapsto\langle\psi, S(t) \phi\rangle(t \in \mathbb{R})$ is differentiable and

$$
\frac{d}{d t}\langle\psi, S(t) \phi\rangle=i\left\{\left\langle H e^{-i t H} \psi, S e^{-i t H} \phi\right\rangle-\left\langle S e^{-i t H} \psi, H e^{-i t H} \phi\right\rangle\right\} .
$$

Proof. It follows from the strong commutativity of $K$ with $H$ and the two-variable functional calculus that $e^{i t H} D(K) \cap D(H)=D(K) \cap D(H)$ for all $t \in \mathbb{R}$. Let $f(t):=$ $\langle\psi, S(t) \phi\rangle, F_{\varepsilon}:=\left(e^{-i \varepsilon H}-1\right) / \varepsilon$ and $G_{\varepsilon}:=e^{-i \varepsilon H}-1$ with $\varepsilon \in \mathbb{R} \backslash\{0\}$. Then

$$
\begin{aligned}
\frac{f(t+\varepsilon)-f(t)}{\varepsilon}= & \left\langle F_{\varepsilon} e^{-i t H} \psi, S e^{-i t H} G_{\varepsilon} \phi\right\rangle+\left\langle F_{\varepsilon} e^{-i t H} \psi, S e^{-i t H} \phi\right\rangle \\
& +\left\langle S e^{-i t H} \psi, e^{-i t H} F_{\varepsilon} \phi\right\rangle .
\end{aligned}
$$

The first term on the right hand side is estimated as follows:

$$
\begin{aligned}
\left|\left\langle F_{\varepsilon} e^{-i t H} \psi, S e^{-i t H} G_{\varepsilon} \phi\right\rangle\right| & \leq\left\|F_{\varepsilon} \psi\right\|\left(a\left\|K e^{-i t H} G_{\varepsilon} \phi\right\|+b\left\|G_{\varepsilon} \phi\right\|\right) \\
& =\left\|F_{\varepsilon} \psi\right\|\left(a\left\|G_{\varepsilon} K \phi\right\|+b\left\|G_{\varepsilon} \phi\right\|\right),
\end{aligned}
$$

where, in the last step, we have used the strong commutativity of $K$ and $H$. Note that $F_{\varepsilon} \psi \rightarrow-i H \psi$ and $G_{\varepsilon} \phi \rightarrow 0$ strongly as $\varepsilon \rightarrow 0$. Hence

$$
\lim _{\varepsilon \rightarrow 0}\left\langle F_{\varepsilon} e^{-i t H} \psi, S e^{-i t H} G_{\varepsilon} \phi\right\rangle=0
$$

and (A.2) follows.

\section{B Ground States of Self-adjoint Operators}

In this section we establish two abstract theorems on existence of ground states of a selfadjoint operator on an abstract Hilbert space. They reveal general structures of methods used in previous papers $[7,12,13]$ to prove the existence of ground states of particle-field interaction models. 


\section{B.1 Existence of a Ground State of a Self-adjoint Operator}

For a self-adjoint operator $S$ on a Hilbert space $\mathcal{X}$, we denote the form domain of $S$ by $Q(S)$ :

$$
Q(S):=\left\{\psi \in \mathcal{X}\left|\int_{\mathbb{R}}\right| \lambda \mid d\left\|E_{S}(\lambda) \psi\right\|^{2}<\infty\right\}=D\left(|S|^{1 / 2}\right),
$$

where $E_{S}(\cdot)$ denotes the spectral measure of $S$ (see Section 2 for notations). For $\psi, \phi \in$ $Q(S)$, we define $\langle\psi, S \phi\rangle$ by

$$
\langle\psi, S \phi\rangle:=\int_{\mathbb{R}} \lambda d\left\langle\psi, E_{S}(\lambda) \phi\right\rangle .
$$

For symmetric operators $A, B$ and a subspace $D \subset D(A) \cap D(B)$, we mean by " $A \leq B$ on $D$ " that $\langle\psi, A \psi\rangle \leq\langle\psi, B \psi\rangle$ for all $\psi \in D$.

Let $\mathcal{H}$ and $\mathcal{K}$ be separable Hilbert spaces. Let $A$ and $B$ be self-adjoint operators on $\mathcal{H}$ and $\mathcal{K}$ respectively. We assume the following:

Hypothesis A. The operator $A$ is bounded from below and $B$ is nonnegative with $E_{0}(B)=0$.

We set

$$
T_{0}:=A \otimes I+I \otimes B,
$$

which is self-adjoint and bounded from below by $E_{0}(A)$.

For a sesquilinear form $Z$ on a Hilbert space, we denote its form domain by $Q(Z)$.

Let $Z$ be a symmemtric sesquilinear form on $\mathcal{H} \otimes \mathcal{K}$ obeying the following conditions:

(i) $Q(Z) \supset Q(I \otimes B)$;

(ii) There exist constants $a \in[0,1)$ and $b \geq 0$ such that, for all $\psi \in Q(I \otimes B)$,

$$
|Z(\psi, \psi)| \leq a\langle\psi, I \otimes B \psi\rangle+b\|\psi\|^{2} .
$$

Lemma B.1 Assume Hypothesis $A$ and and let $Z$ be as above. Then there exists a unique self-adjoint operator $T$ on $\mathcal{H} \otimes \mathcal{K}$ such that $Q(T)=Q\left(T_{0}\right)$ and

$$
\langle\psi, T \phi\rangle=\left\langle\psi, T_{0} \phi\right\rangle+Z(\psi, \phi), \quad \psi, \phi \in Q\left(T_{0}\right) .
$$

$T$ is bounded from below by $E_{0}(A)-b$ and every domain of essential self-adjointness for $T_{0}$ is a form core for $T$.

Proof. Let $\hat{A}:=A-E_{0}(A)$, which is nonnegative. By the present assumption, we have for all $\psi \in Q\left(T_{0}\right)$,

$$
|Z(\psi, \psi)| \leq a\langle\psi,(\hat{A} \otimes I+I \otimes B) \psi\rangle+b\|\psi\|^{2} .
$$

Note that $\hat{A} \otimes I+I \otimes B \geq 0$. Hence we can apply the KLMN theorem [16, Theorem X.17] to conclude that there exists a unique self-adjoint operator $T^{\prime}$ on $\mathcal{H} \otimes \mathcal{K}$ such that $Q\left(T^{\prime}\right)=Q(\hat{A} \otimes I+I \otimes B)=Q\left(T_{0}\right)$ and $T^{\prime}=\hat{A} \otimes I+I \otimes B+Z$ in the sense of sesquilinear form on $Q\left(T_{0}\right)$ with $T^{\prime} \geq-b$. Then the operator $T$ defined by $T:=T^{\prime}+E_{0}(A)$ is the desired one. 
Lemma B.2 Under the same hypothesis as in Lemma B.1,

$$
\left|E_{0}(T)-E_{0}(A)\right| \leq b
$$

Proof. By the variational principle and Lemma B.1, we have

$$
E_{0}(T) \geq E_{0}(A)-b
$$

On the other hand, for all $f \in D(A)$ and $g \in D(B)$ with $\|f\|=1$ and $\|g\|=1$, we have

$$
E_{0}(T) \leq(f, A f)+(1+a)(g, B g)+b,
$$

which, together with the variational principle, implies that $E_{0}(T) \leq E_{0}(A)+b$, where we have used the condition $E_{0}(B)=0$. Hence (B.3) follows.

We set

$$
\Sigma:=\inf \sigma_{\mathrm{ess}}(A)
$$

and, for $s>0$,

$$
\beta(s):=\frac{E_{0}(T)-E_{0}(A)+b+s}{1-a} .
$$

By (B.3), we have

$$
\beta(s) \geq \frac{s}{1-a}>0 .
$$

Theorem B.3 Assume Hypothesis $A$ and let $Z$ be as above. Suppose that

$$
\Sigma-E_{0}(T)>b
$$

and, for some $s_{0}>0, \operatorname{Ran}\left(E_{B}\left(\left[0, \beta\left(s_{0}\right)\right]\right)\right.$ is finite dimensional. Let $m$ be a constant such that

$$
\begin{aligned}
& \Sigma-E_{0}(T)>m+b, \\
& 0<m<s_{0} .
\end{aligned}
$$

Then $T$ has purely discrete spectrum in the interval $\left[E_{0}(T), E_{0}(T)+m\right)$. In particular, $T$ has a ground state.

Remark B.1 By Lemma B.2, condition (B.8) is satisfied if

$$
\Sigma-E_{0}(A)>2 b .
$$

Proof. Let

$$
\hat{A}:=A-E_{0}(A), \quad T_{m}:=T-E_{0}(T)-m .
$$

Then we have on $D\left(T_{0}\right)$

$$
\begin{aligned}
T_{m} & =\hat{A} \otimes I+I \otimes B+Z+E_{0}(A)-E_{0}(T)-m \\
& \geq \hat{A} \otimes I+I \otimes(1-a) B-\alpha_{0},
\end{aligned}
$$


where

$$
\alpha_{0}:=E_{0}(T)-E_{0}(A)+b+m \geq m .
$$

Since we have (B.9)

$$
\alpha_{0}<\Sigma-E_{0}(A),
$$

one can take a constant $\delta>0$ such that $\alpha_{0} \leq \delta<\Sigma-E_{0}(A)$. Let $P_{\delta}:=E_{\hat{A}}([0, \delta])$ and $P_{\delta}^{\perp}:=I-P_{\delta}=E_{\hat{A}}((\delta, \infty))$, so that $P_{\delta}+P_{\delta}^{\perp}=I$. Then we have

$$
\begin{aligned}
& \hat{A} \otimes I+I \otimes(1-a) B-\alpha_{0} \\
= & P_{\delta} \hat{A} \otimes I+P_{\delta}^{\perp} \hat{A} \otimes I+P_{\delta} \otimes(1-a) B+P_{\delta}^{\perp} \otimes(1-a) B-\alpha_{0} P_{\delta} \otimes I-\alpha_{0} P_{\delta}^{\perp} \otimes I .
\end{aligned}
$$

Note that

$$
P_{\delta} \hat{A} \otimes I \geq 0, \quad P_{\delta}^{\perp} \hat{A} \otimes I \geq \delta P_{\delta}^{\perp} \otimes I, \quad P_{\delta}^{\perp} \otimes(1-a) B \geq 0 .
$$

Hence

$$
\begin{aligned}
\hat{A} \otimes I+I \otimes(1-a) B-\alpha_{0} & \geq\left(\delta-\alpha_{0}\right) P_{\delta}^{\perp} \otimes I+P_{\delta} \otimes\left[(1-a) B-\alpha_{0}\right] \\
& \geq\left(P_{\delta} \otimes\left[(1-a) B-\alpha_{0}\right],\right.
\end{aligned}
$$

where we have used the condition $\delta \geq \alpha_{0}$. Hence we have on $D\left(T_{0}\right)$

$$
T_{m} \geq P_{\delta} \otimes\left[(1-a) B-\alpha_{0}\right] \geq P_{\delta} \otimes\left[(1-a) B-\alpha_{0}\right]_{-},
$$

where $\left[(1-a) B-\alpha_{0}\right]_{-}$means the negative part of $(1-a) B-\alpha_{0}$. Let $J_{m}:=E_{T_{m}}([-m, 0))$.

(i) The case where $\operatorname{Ran}\left(J_{m}\right)$ is finite dimensional. In this case $T_{m}$ has a purely discrete spectrum in $[-m, 0)$. This means that the spectrum of $T$ in $\left[E_{0}(T), E_{0}(T)+m\right)$ is purely discrete. In particular $T$ has a ground state.

(ii) The case where $\operatorname{Ran}\left(J_{m}\right)$ is infinite dimensional. Note that

$$
\left[(1-a) B-\alpha_{0}\right]_{-}=E_{B}\left([0, \beta(m))\left[(1-a) B-\alpha_{0}\right] E_{B}([0, \beta(m)) .\right.
$$

By condition (B.10) and the present assumption, $\operatorname{Ran}\left(E_{B}([0, \beta(m)))\right)$ is finite dimensional. Hence $\left[(1-a) B-\alpha_{0}\right]_{-}$is trace class. Therefore $P_{\delta} \otimes\left[(1-a) B-\alpha_{0}\right]_{-}$is trace class. Let $\left\{\psi_{n}\right\}_{n=1}^{\infty}$ be a complete orthonormal system of $\operatorname{Ran}\left(J_{m}\right)$. Then, for all $N \in \mathbb{N}$,

$$
\begin{aligned}
0 & \geq \sum_{n=1}^{N}\left\langle\psi_{n}, T_{m} \psi_{n}\right\rangle \geq \sum_{n=1}^{N}\left\langle\psi_{n}, P_{\delta} \otimes\left[(1-a) B-\alpha_{0}\right]_{-} \psi_{n}\right\rangle \\
& \geq \operatorname{Tr}\left\{P_{\delta} \otimes\left[(1-a) B-\alpha_{0}\right]_{-}\right\}
\end{aligned}
$$

Hence $\sum_{n=1}^{N}\left\langle\psi_{n}, T_{m} \psi_{n}\right\rangle$ is convergent as $N \rightarrow \infty$, which implies $J_{m} T_{m} J_{m}$ is trace class and hence it is compact. Thus $T_{m}$ has purely discrete spectrum in $[-m, 0)$, which implies that $T$ has purely discrete spectrum in $\left[E_{0}(T), E_{0}(T)+m\right)$. In particular $T$ has a ground state. 


\section{B.2 A Limit Theorem on Ground States}

Let $K$ be a self-adjoint operator on $\mathcal{H}$ bounded from below and $B$ be a nonnegative selfadjoint operator on $\mathcal{K}$ with $E_{0}(B)=0$. Let $C$ be a symmetric operator on $\mathcal{H} \otimes \mathcal{K}$ with $D(K \otimes I) \cap D(I \otimes B) \subset D(C)$ such that

$$
H:=K \otimes I+I \otimes B+C
$$

is self-adjoint and bounded from below.

Let

$$
\Sigma:=\inf \sigma_{\mathrm{ess}}(K)
$$

Hypothesis (B.1). $\Sigma>E_{0}(K)$.

Hypothesis (B.2). There are a family $\left\{K_{m}\right\}_{m \in\left(0, m_{0}\right]}$ of symmetric operators on $\mathcal{H}$ with $D(K) \subset \cap_{m \in\left(0, m_{0}\right]} D\left(K_{m}\right)$ and a family $\left\{B_{m}\right\}_{m \in\left(0, m_{0}\right]}$ of nonnegative self-adjoint operators on $\mathcal{K}$ with $E_{0}\left(B_{m}\right)=0$ such that the following hold:

(i) There exists a constant $c_{m}>0$ such that, for all $u \in D(K)$,

$$
\left\|\left(K-K_{m}\right) u\right\| \leq c_{m}(\|K u\|+\|u\|)
$$

and $\lim _{m \rightarrow 0} c_{m}=0$.

(ii) There exists a non-zero vector $\psi_{0}$ such that, for all $m \in\left(0, m_{0}\right], B_{m} \psi_{0}=0$. We denote the orthogonal projection onto the one-dimensional subspace $\left\{\alpha \psi_{0} \mid \alpha \in \mathbf{C}\right\}$ by $P_{0}$.

(iii) For each $m \in\left(0, m_{0}\right], D\left(K_{m} \otimes I\right) \cap D\left(I \otimes B_{m}\right) \subset D(C)$ and the operator

$$
H_{m}:=K_{m} \otimes I+I \otimes B_{m}+C
$$

is self-adjoint and bounded from below.

(iv) There exists a dense subspace $D \subset\left[\cap_{m \in\left(0, m_{0}\right]} D\left(B_{m}\right)\right] \cap D(B)$ such that, for all $\psi \in D, \lim _{m \rightarrow 0} B_{m} \psi=B \psi$ and $D(K) \otimes_{\text {alg }} D$ is a core of $H$, where $\otimes_{\text {alg }}$ means algebraic tensor product.

For an orthogonal projection $P$ on a Hilbert space, we set

$$
P^{\perp}:=I-P \text {. }
$$

Theorem B.4 Assume Hypotheses (B.1) and (B.2). Suppose that

$$
\inf _{m \in\left(0, m_{0}\right]} E_{0}\left(H_{m}\right)>-\infty, \quad \sup _{m \in\left(0, m_{0}\right]} E_{0}\left(H_{m}\right)<\Sigma .
$$

Suppose that, for all $m \in\left(0, m_{0}\right], H_{m}$ has a ground state $\Psi_{m}$ with $\left\|\Psi_{m}\right\|=1$ and there exists a constant $\delta<1$ independent of $m \in\left(0, m_{0}\right]$ such that

$$
\frac{1}{\left(\Sigma-E_{0}\left(H_{m}\right)\right)^{2}}\left\|C \Psi_{m}\right\|^{2}+\left\|I \otimes P_{0}^{\perp} \Psi_{m}\right\|^{2}<\delta .
$$


Then there exists a subsequence $\left\{\Psi_{m_{j}}\right\}_{j=1}^{\infty}$ with

$$
m_{1}>m_{2}>\cdots>m_{j}>m_{j+1}>\cdots, \quad \lim _{j \rightarrow \infty} m_{j}=0
$$

such that the weak limit $\Psi_{0}:=\mathrm{w}-\lim _{j \rightarrow \infty} \Psi_{m_{j}}$ is a ground state of $H$.

Proof. We divide the proof of this theorem into two steps.

(1) By Hypothesis (B.2)-(i), there exists a positive constant $\varepsilon_{0}<m_{0}$ such that, for all $0<m<\varepsilon_{0}, c_{m}<1$. Then, by the Kato-Rellich theorem, $K_{m}=K+\left(K_{m}-K\right)$ is self-adjoint with $D\left(K_{m}\right)=D(K)$ and bounded from below. We can take a constant $\xi$ such that $\max \left\{\sup _{m \in\left(0, m_{0}\right]} E_{0}\left(H_{m}\right), E_{0}(K)\right\}<\xi<\Sigma$ and

$$
\frac{1}{\left(\xi-E_{0}\left(H_{m}\right)\right)^{2}}\left\|C \Psi_{m}\right\|^{2}+\left\|I \otimes P_{0}^{\perp} \Psi_{m}\right\|^{2} \leq \delta .
$$

Let

$$
P_{K}:=E_{K}\left(\left[E_{0}(K), \xi\right]\right) .
$$

Then, by Hypothesis (B.1), $\operatorname{dim} \operatorname{Ran} P_{K}<\infty$.

Let $K_{m}(\beta):=K+\beta L_{m}$ with $L_{m}:=\left(K_{m}-K\right) / c_{m}$ and $\beta \in \mathbb{C}$. Since $L_{m}$ is relatively bounded with respect to $K$, it follows from [17, p.16, Lemma] that $K_{m}(\beta)$ is an analytic family of type $(\mathrm{A})$ near $\beta=0$. Hence it is an analytic family in the sense of Kato $[17$, p.17, Theorem XII.9] and $K_{m}(\beta)$ is self-adjoint for real $\beta$ with $|\beta|$ sufficiently small. We define

$$
Q_{m}(\beta):=E_{K_{m}(\beta)}\left(\left[E_{0}(K), \xi\right]\right)
$$

and

$$
Q_{m}:=E_{K_{m}}\left(\left[E_{0}(K), \xi\right]\right) .
$$

Then $Q_{m}(\beta)$ is analytic near $\beta=0$. In particular,

$$
\lim _{\beta \rightarrow 0}\left\|Q_{m}(\beta)-P_{K}\right\|=0
$$

and hence $\operatorname{dim} \operatorname{Ran} Q_{m}(\beta)=\operatorname{dim} \operatorname{Ran} P_{K}<\infty$ for all sufficiently small $|\beta|$. Note that $K_{m}\left(c_{m}\right)=K_{m}$. Therefore, for every $\varepsilon>0$, there exists a constant $\eta_{0}>0$ such that, for all $m \in\left(0, \eta_{0}\right)$,

$$
\left\|Q_{m}-P_{K}\right\|<\varepsilon
$$

and $\operatorname{dim} \operatorname{Ran} Q_{m}=\operatorname{dim} \operatorname{Ran} P_{K}$.

(2) By the weak compactness of the unit ball of a Hilbert space and condition (B.15), there exists a subsequence $\left\{\Psi_{m_{j}}\right\}_{j=1}^{\infty}\left(m_{1}>m_{2}>\cdots>m_{j}>m_{j+1}>\cdots, \lim _{j \rightarrow \infty} m_{j}=0\right)$ such that the weak limit $\Psi_{0}:=\mathrm{w}-\lim _{j \rightarrow \infty} \Psi_{m_{j}}$ and $E_{0}:=\lim _{j \rightarrow \infty} E_{0}\left(H_{m_{j}}\right)$ exist. By Hypothesis (B.2)-(iii), we have $\lim _{m \rightarrow 0} H_{m} \Psi=H \Psi$ for all $\Psi \in D(K) \otimes_{\text {alg }} D$. Hence, by an applicaiton of $\left[4\right.$, Lemma 4.9], if we show that $\Psi_{0} \neq 0$, then we can conclude that $\Psi_{0}$ is a ground state with $E_{0}=E_{0}(H)$.

We have $\operatorname{dim} \operatorname{Ran} P_{0}=1$. Hence, to show that $\Psi_{0} \neq 0$, we need only to prove

$$
\left\langle\Psi_{m}, P_{K} \otimes P_{0} \Psi_{m}\right\rangle \geq 1-\delta^{\prime}
$$


with a constant $\delta^{\prime}<1$ independent of $m$. Then, passing to the subsequence $\left\{\Psi_{m_{j}}\right\}_{j}$ and taking the limit $j \rightarrow \infty$, we obtain $\left\langle\Psi_{0}, P_{K} \otimes P_{0} \Psi_{0}\right\rangle \geq 1-\delta^{\prime}>0$, which implies that $\Psi_{0} \neq 0$.

To prove (B.19), we first prove

$$
\left\langle\Psi_{m}, Q_{m} \otimes P_{0} \Psi_{m}\right\rangle \geq 1-\delta .
$$

Then, by (B.18), we obtain (B.19) for all $m<\eta_{0}$ with $\delta^{\prime}=\delta+\varepsilon<1$ and hence the proof is completed.

Now we note that (B.20) is equivalent to

$$
\left\langle\Psi_{m},\left(Q_{m}^{\perp} \otimes P_{0}+I \otimes P_{0}^{\perp}\right) \Psi_{m}\right\rangle \leq \delta .
$$

This is seen by using the identity $1=\left\langle\Psi_{m},\left(Q_{m}+Q_{m}^{\perp}\right) \otimes\left(P_{0}+P_{0}^{\perp}\right) \Psi_{m}\right\rangle$. We prove (B.21). We have

$$
\left(Q_{m}^{\perp} \otimes P_{0}\right) H_{m}=Q_{m}^{\perp} K_{m} \otimes P_{0}+Q_{m}^{\perp} \otimes P_{0} C .
$$

Hence

$$
\begin{aligned}
0 & =\left(Q_{m}^{\perp} \otimes P_{0}\right)\left(H_{m}-E_{0}\left(H_{m}\right)\right) \Psi_{m} \\
& =\left(Q_{m}^{\perp}\left(K_{m}-E_{0}\left(H_{m}\right)\right) \otimes P_{0}\right) \Psi_{m}+\left(Q_{m}^{\perp} \otimes P_{0}\right) C \Psi_{m}
\end{aligned}
$$

which implies that

$$
\begin{aligned}
\left\langle\Psi_{m},\left(Q_{m}^{\perp} \otimes P_{0}\right) C \Psi_{m}\right\rangle & =-\left\langle\Psi_{m}, Q_{m}^{\perp}\left(K_{m}-E_{0}\left(H_{m}\right)\right) \otimes P_{0} \Psi_{m}\right\rangle \\
& \leq-\left(\xi-E_{0}\left(H_{m}\right)\right)\left\langle\Psi_{m}, Q_{m}^{\perp} \otimes P_{0} \Psi_{m}\right\rangle .
\end{aligned}
$$

Hence

$$
\begin{aligned}
\left\langle\Psi_{m}, Q_{m}^{\perp} \otimes P_{0} \Psi_{m}\right\rangle & \leq-\frac{1}{\xi-E_{0}\left(H_{m}\right)}\left\langle\Psi_{m},\left(Q_{m}^{\perp} \otimes P_{0}\right) C \Psi_{m}\right\rangle \\
& \leq \frac{1}{\xi-E_{0}\left(H_{m}\right)}\left\|Q_{m}^{\perp} \otimes P_{0} \Psi_{m}\right\|\left\|C \Psi_{m}\right\| .
\end{aligned}
$$

Hecne

$$
\left\langle\Psi_{m}, Q_{m}^{\perp} \otimes P_{0} \Psi_{m}\right\rangle \leq \frac{1}{\left(\xi-E_{0}\left(H_{m}\right)\right)^{2}}\left\|C \Psi_{m}\right\|^{2},
$$

which, together with (B.17), yields (B.21).

\section{Acknowledgements}

This work was completed during the stay of the first author (A. A.) at the Erwin Schrödinger International Institute for Mathematical Physics (ESI) in the autumn, 2002. A. A. would like to thank Professor H. Grosse for giving him an opportunity to participate in the ESI program: Noncommutative Geometry and Quantum Field Theory and warm hospitality. A. A. also acknowledges the support given by the ESI. This work was supported in part also by the Grant-In-Aid No.13440039 for scientific research from the Japan Society for Promotion of Science. 


\section{References}

[1] A. Arai, Self-adjointness and spectrum of Hamiltonians in nonrelativistic quantum electrodynamics, J. Math. Phys. 22 (1981), 534-537.

[2] A. Arai, An asymptotic analysis and its application to the nonrelativistic limit of the Pauli-Fierz and a spin-boson model, J. Math. Phys. 31 (1990), 2653-2663.

[3] A. Arai, Fock Spaces and Quantum Fields, Nippon-Hyouronsha, Tokyo, 2000 (in Japanese).

[4] A. Arai and M. Hirokawa, On the existence and uniqueness of ground states of a generalized spin-boson model, J. Funct. Anal. 151 (1997), 455-503.

[5] A. Arai and M. Hirokawa, Ground states of a general class of quantum field Hamiltonians, Rev. Math. Phys. 8 (2000), 1085-1135.

[6] A. Arai, M. Hirokawa and F. Hiroshima, On the absence of eigenvectors of Hamiltonians in a class of massless quantum field models without infrared cutoff, J. Funct. Anal. 168 (1999), 470-497.

[7] V. Bach, J. Fröhlich and I. M. Sigal, Quantum elecrodynamics of confined nonrelativistic particles, Adv. Math. 137 (1998), 299-395.

[8] I. Catto and C. Hainzl, Self-energy of one electron in non-relativistic QED, mathph/0207036, 2002.

[9] T. Chen, V. Vougalter and S. A. Vugalter, The increase of binding energy and enhanced binding in non-relativistic QED, math-ph/0209062, 2003.

[10] C. Hainzl, One non-relativistic particle coupled to a photon field, math-ph/0202001, 2002.

[11] C. Hainzl, V. Vougalter and S. A. Vugalter, Enhanced binding in non-relativistic QED, mp_arc 01-455, 2001.

[12] F. Hiroshima, Ground states of a model in nonrelativistic quantum electrodynamics I, J. Math. Phys. 40 (1999), 6209-6222.

[13] F. Hiroshima, Analysis of ground states of atoms interacting with a quantized radiation field, preprint, 2002.

[14] F. Hiroshima and H. Spohn, Enhanced binding through coupling to a quantum field, Ann. Henri Poincaré 2 (2001), 1159-1187.

[15] M. Reed and B. Simon, Methods of Modern Mathematical Physics Vol. I, Academic Press, New York, 1972.

[16] M. Reed and B. Simon, Methods of Modern Mathematical Physics Vol. II, Academic Press, New York, 1975. 
[17] M. Reed and B. Simon, Methods of Modern Mathematical Physics Vol. IV, Academic Press, New York, 1978. 